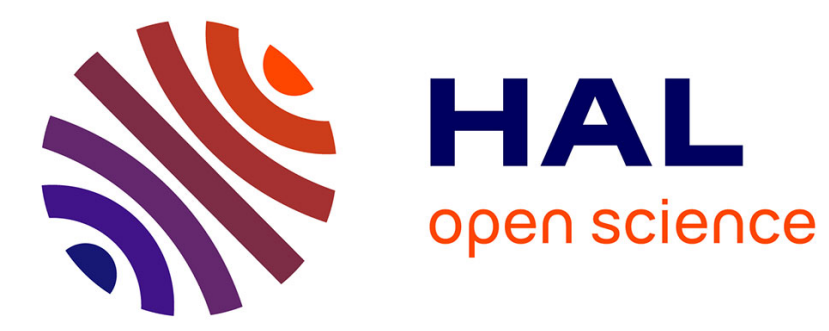

\title{
Les avions français de recherche atmosphérique et de télédétection: bilan et perspectives
}

Jean-Pierre Chalon, M. André, J.-L. Brenguier, A. Druilhet, J.-F. Gayet, Pierre Henri Flamant, B. Guillemet, Danièle Hauser, Yann H. Kerr, Jacques Pelon, et al.

\section{To cite this version:}

Jean-Pierre Chalon, M. André, J.-L. Brenguier, A. Druilhet, J.-F. Gayet, et al.. Les avions français de recherche atmosphérique et de télédétection: bilan et perspectives. La Météorologie, 1998, 8 (22), pp.14-44. 10.4267/2042/47046 . hal-02053305

\section{HAL Id: hal-02053305 https://hal.uca.fr/hal-02053305}

Submitted on 1 Mar 2019

HAL is a multi-disciplinary open access archive for the deposit and dissemination of scientific research documents, whether they are published or not. The documents may come from teaching and research institutions in France or abroad, or from public or private research centers.
L'archive ouverte pluridisciplinaire HAL, est destinée au dépôt et à la diffusion de documents scientifiques de niveau recherche, publiés ou non, émanant des établissements d'enseignement et de recherche français ou étrangers, des laboratoires publics ou privés. 


\title{
LES AVIONS FRANÇAIS DE RECHERCHE ATMOSPHÉRIQUE ET DE TÉLÉDÉTECTION
}

\author{
BILAN ET PERSPECTIVES
}

\author{
J.-P. Chalon ${ }^{(1)}$, M. André(1), J.-L. Brenguier ${ }^{(1)}$, A. Druilhet ${ }^{(2)}$, \\ J.-F. Gayet ${ }^{(3)}$, P. Flamant ${ }^{(4)}$, B. Guillemet ${ }^{(3)}$, D. Hauser ${ }^{(5)}$, \\ Y. Kerr ${ }^{(6)}$, J. Pelon ${ }^{(7)}$, M. Ravaut ${ }^{(8)}$ et D. Tanré( ${ }^{(9)}$ \\ (1) Météo-France \\ École nationale de la météorologie (ENM) et Centre national de \\ recherches météorologiques (CNRM, Game-URA 1357 du CNRS) \\ 42, avenue Gaspard-Coriolis, 31057 Toulouse Cedex \\ (2) Laboratoire d'aérologie (LA) \\ CNRS et université Paul Sabatier \\ Observatoire Midi-Pyrénées, Toulouse \\ (3) Laboratoire de météorologie physique (LAMP) \\ CNRS et université de Clermont-Ferrand 2 \\ Observatoire de physique du globe de Clermont-Ferrand, Aubière \\ (4) Laboratoire de météorologie dynamique (LMD) du CNRS \\ École polytechnique, Palaiseau \\ (5) Centre d'étude des environnements terrestre et planétaires (CETP) \\ CNRS et université de Versailles - Saint-Quentin, Vélizy \\ (6) Centre d'études spatiales de la biosphère (Cesbio) \\ Cnes, CNRS et université Paul Sabatier, Toulouse \\ (7) Service d'aéronomie (SA) du CNRS \\ Université Pierre et Marie Curie, Paris \\ (8) Institut national des sciences de I'univers (Insu) du CNRS, Paris \\ (9) Laboratoire d'optique atmosphérique (LOA) \\ CNRS et université des sciences et techniques de Lille, Villeneuve-d'Ascq
}

Ndlr : quelques-unes des illustrations de cet article sont publiées en couleurs p. 85 et 86.

RÉSUMÉ Alors que nous sommes en train de planifier le remplacement des avions français utilisés par la communauté scientifique en recherche atmosphérique et en télédétection, nous avons voulu présenter un état des lieux montrant les moyens disponibles (les avions, leur instrumentation et leurs filières de traitement des données) et quelques exemples de travaux scientifiques ayant bénéficié de ces outils.

La dernière partie de l'article est tournée vers l'avenir. Elle présente une synthèse des travaux prospectifs réalisés par le Conseil scientifique des avions de recherche atmosphérique et de télédétection pour évaluer les besoins futurs de la communauté scientifique et en déduire les qualités et les performances requises pour les futurs avions de recherche et leur instrumentation. 


\section{ABSTRACT The French aircraft for atmospheric research and remote sensing: status and prospects}

We are planning to replace the French airplanes used for atmospheric research and remote sensing, and we wish to describe some of the current systems (aircraft, instrumentation and data processing) and give some examples of their use.

The last part of the article looks the future. It is a summary of proposals made by the Scientific Committee for Atmospheric and Remote Sensing Research Aircraft of future needs, and infers the specifications required for the future aircraft and their instrumentation.

La compréhension des phénomènes météorologiques et climatiques nécessite une observation détaillée des processus physiques et chimiques qui se produisent dans l'atmosphère et à la surface terrestre (sols, végétation, océan). Dans le domaine des sciences atmosphériques, l'avion est ainsi devenu un outil indispensable pour affiner les observations à très grande échelle obtenues grâce aux réseaux météorologiques et aux satellites. Il permet de mesurer, directement au cœur des phénomènes étudiés, des paramètres tels que la température, l'humidité, le vent, le rayonnement, la granulométrie des nuages et des précipitations, et d'effectuer des prélèvements in situ (échantillons d'aérosols, d'air ou de précipitations) pour en faire l'analyse chimique et physique. L'utilisation d'équipements de télédétection comme les lidars et les radars embarqués permet d'obtenir des informations précieuses sur la structure tridimensionnelle de l'atmosphère.

Au cours des dernières années, la perception du système climatique a orienté les recherches vers une approche globale de l'étude de ce système, régi par les interactions entre l'atmosphère, l'océan, la cryosphère, les terres émergées et la biosphère. Ainsi, l'observation à distance des surfaces continentales et océaniques est-elle devenue une nécessité de plus en plus évidente pour répondre aux besoins des scientifiques cherchant à comprendre et à surveiller les changements globaux de notre planète, comme à ceux des gestionnaires des ressources terrestres (cartographes, géologues, agronomes, urbanistes...). Dans ce contexte, les instruments aéroportés, capables d'acquérir des images dans de multiples bandes spectrales, fournissent des informations précieuses mais permettent aussi de préparer les futures missions spatiales et donnent aux utilisateurs le moyen de développer les méthodes d'interprétation de ces images. L'avion est aussi une plate-forme idéale pour larguer des sondes permettant d'obtenir des profils verticaux de l'atmosphère ou de l'océan, ainsi que des bouées capables de fournir des renseignements sur les courants marins et les échanges océan-atmosphère.

DESCRIPTION DES MOYENS ACTUELS DE LA COMMUNAUTÉ SCIENTIFIQUE NATIONALE

En France, trois avions de recherche instrumentés sont consacrés à l'étude de l'atmosphère et des surfaces océaniques ou continentales : un Merlin IV et un Piper Aztec mis en œuvre par Météo-France au Centre d'aviation météorologique du CNRM, un Fokker 27 baptisé Arat (Avion de recherche atmosphérique et de télédétection), exploité par le Centre national d'études spatiales (Cnes), l'Institut géographique national (IGN), l'Institut national des sciences de l'univers (Insu) du CNRS et Météo-France, et mis en œuvre par le Service des activités aériennes de l'IGN à Creil. Les limitations d'emport de ces avions ont aussi conduit à installer des équipement français sur des avions étrangers : antenne double-faisceau pour le radar Doppler de queue d'un avion P3 de la NOAA, radar Doppler franco-américain Eldora-Astraia sur l'avion Electra du NCAR, lidar Doppler Wind sur l'avion Falcon du DLR allemand. En dehors de ces avions, la Caravelle et le Transall du Centre d'essais en vol (CEV) ont été utilisés de façon épisodique, principalement pour l'étude des champs d'ozone à grande échelle (campagnes Stratoz en 1978, 1980 et 1984, campagnes Tropoz en 1985 et 1991) et du foudroiement des avions dans les orages (campagne Landes-Fronts 84). Il convient aussi de mentionner le programme Mozaic, lancé en 1993 pour une meilleure connaissance des champs d'ozone stratosphérique. Ce programme permet de recueillir des mesures de concentration d'ozone et de vapeur d'eau, de 
vent et de température à l'occasion d'environ 1500 vols commerciaux effectués chaque année par les cinq Airbus A340 équipés par Air France, Sabena, Lufthansa et Austrian Airlines. Enfin, la possibilité de louer à l'IGN un Falcon 20 pouvant voler à plus de $11000 \mathrm{~m}$ d'altitude avec une autonomie de 3 heures 45 , pour une charge scientifique de $500 \mathrm{~kg}$, est à l'étude.

\section{Les avions}

L'Arat

Le Fokker 27 (Arat) est un biturbopropulseur profondément modifié pour recevoir une instrumentation scientifique variée. Il est autorisé à voler en conditions givrantes. Son autonomie en vol est comprise entre 3 heures et 5 heures 30 suivant la configuration adoptée. Son plafond est limité à 5800 mètres. L'Arat est équipé d'une centrale à inertie Uliss 45 (Sagem) et de deux récepteurs GPS qui délivrent les données d'attitude, de navigation et de temps. La rusticité de l'avion, la taille du fuselage et la disponibilité de $30 \mathrm{kVA}$ entièrement réservés aux expériences scientifiques font de l'Arat une plate-forme très polyvalente.

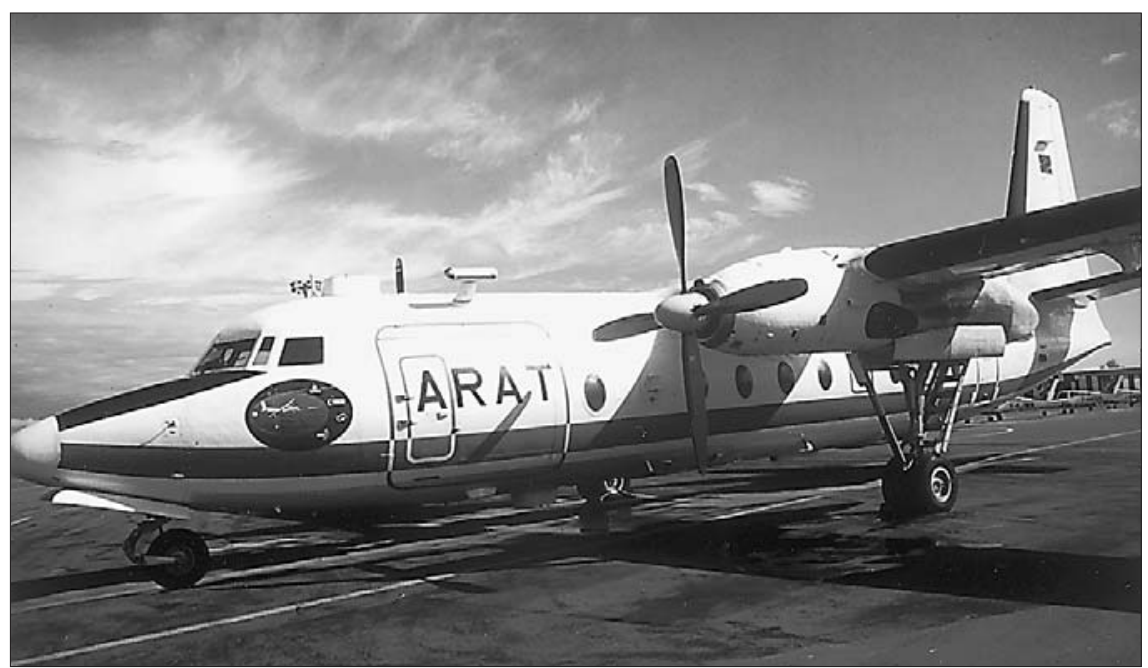

L'avion Arat au sol. (Photo Météo-France, P. Taburet)

En version atmosphère, des mesures in situ peuvent être associées à des mesures de télédétection par lidar. L'instrumentation permet d'accéder aux caractéristiques turbulentes des champs thermodynamiques (vitesse, température et teneur en vapeur d'eau) dans la moyenne et la basse troposphère. La résolution spatiale est de l'ordre de $10 \mathrm{~m}$. Les mesures sont effectuées à l'extrémité d'une perche de $6 \mathrm{~m}$, dans une région où les écoulements sont peu sensibles aux perturbations aérodynamiques engendrées par l'avion. L'Arat est également équipé pour effectuer des mesures radiatives (composantes montante et descendante des flux radiatifs) dans des bandes ultraviolette, visible, rouge et infrarouge. L'étude de la microphysique des nuages est abordée grâce à un ensemble de sondes PMS (Particule Measuring Systems) disposées sous les ailes ou autour du fuselage. Celles-ci fournissent la distribution granulométrique des noyaux de condensation, des cristaux de glace, des gouttes de nuage et de pluie. Actuellement, de gros efforts sont en cours pour associer à ce système un ensemble de mesures permettant de caractériser la chimie de la basse troposphère : fluctuations turbulentes de la concentration en ozone et en aérosols, concentrations moyennes de divers composés tels que l'ozone, $\mathrm{CO}, \mathrm{CO}_{2}$, les oxydes d'azote $\left(\mathrm{NO}, \mathrm{NO}_{2}, \mathrm{NO}_{\mathrm{y}}\right)$, les hydrocarbures non méthaniques, etc. Le spectromètre à diode laser Jetdlag permet la mesure des concentrations en $\mathrm{O}_{3}$, $\mathrm{CO}, \mathrm{CH}_{4}$. Une veine isocinétique donne accès à l'étude physique et chimique des aérosols à partir de filtres impacteurs. Dans cette version, l'Arat peut aussi être équipé du lidar aérosol Leandre 1, du lidar vapeur d'eau Leandre 2 ou du lidar ozone Alto et pourra bientôt accueillir le lidar vent Wind actuellement en cours de développement avec le DLR. Grâce à un ensemble de hublots vers le haut et vers le bas, ces systèmes sont capables de décrire l'atmosphère depuis la surface terrestre jusqu'à la basse stratosphère. 


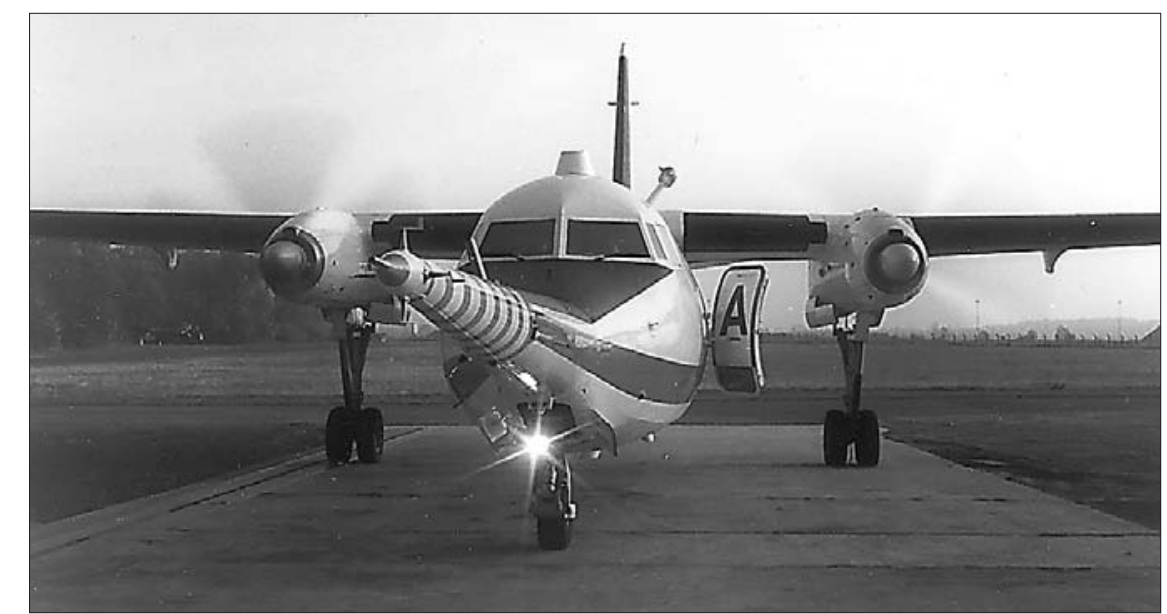

L'avion Arat vu de face, équipé d'une perche instrumentée de 6 mètres. (Photo IGN)

En version télédétection, l'Arat devient une plate-forme aéroportée embarquant des prototypes d'instruments qui, une fois qualifiés, peuvent être utilisés dans des campagnes de mesures scientifiques. Parmi les instruments qui ont été installés sur l'Arat, on peut ainsi citer le radar à synthèse d'ouverture Varan-S, le radiomètre multispectral Push-Broom, le radiomètre profileur Portos, le polarimètre Polder et le radar imageur Sethy.

Le Merlin IV Le Merlin IV est un biturbopropulseur pressurisé, autorisé au vol en conditions givrantes. Suivant la configuration adoptée, son autonomie en vol est comprise entre 3 et 5 heures et son plafond de vol est compris entre $6600 \mathrm{~m}$ et $8700 \mathrm{~m}$. Le Merlin IV est doté d'une centrale de navigation à inertie Uliss 45 (Sagem). Comme l'Arat, il est instrumenté pour les mesures in situ de la moyenne et basse troposphère et effectue l'acquisition des champs thermodynamiques turbulents avec une résolution comparable. Il peut également être équipé de capteurs de mesures radiatives et microphysiques ou d'un système spécifique de prélèvement permettant l'analyse physico-chimique de l'air, de l'eau nuageuse et des précipitations (pollution, pluies acides...). Les mesures thermodynamiques à haute résolution sont réalisées avec un radôme spécialement étudié. Cette technique simple ne nécessite pas de modification de structure importante et conduit à des résultats équivalents à ceux obtenus avec une perche.

Depuis 1993, le Merlin IV est équipé d'un réfractomètre pour mesurer les fluctuations rapides d'indice de réfraction de l'air. Ce dispositif permet d'aborder l'étude de la transmission dans les milieux turbulents et stratifiés, mais aussi d'estimer les fluctuations turbulentes d'humidité avec une précision inégalée. Il a en particulier été utilisé pour la validation de mesures radar et de techniques d'observation en air clair. Ces dernières années, l'équipement de l'avion a évolué vers des mesures plus rapides, tant pour le système d'acquisition des données (paramètres thermodynamiques échantillonnés pour la plupart à $200 \mathrm{~Hz}$ ) que pour les capteurs de mesure de la température (fil fin de tungstène développé au Service des équipements et des techniques instrumentales de la météorologie de Météo-France, thermo-anémomètre sonique développé par le LMD), de la vitesse propre de l'avion (thermo-anémomètre du LMD), de la teneur en eau liquide (diffusiomètre à faisceau laser Gerber), de l'incidence de l'avion (girouette asservie et dégivrée de la société Sextant Avionique) et des fluctuations de pression (variomètre rapide de grande sensibilité de Sextant Avionique).

Le Merlin IV peut aussi être utilisé en version télédétection avec le radar à diffusion Ressac (mesure de l'état de surface de l'océan et du spectre des vagues) mis en œuvre par le CETP. 
À l'avant du Merlin IV, le radôme instrumenté (en noir) est muni de capteurs de pression et d'une centrale à inertie pour mesurer le vent par anémoclinométrie. Juste derrière, la couronne instrumentée (en blanc) porte des capteurs rapides de température et d'humidité.

(Photo Météo-France, P. Taburet)

Le Piper Aztec

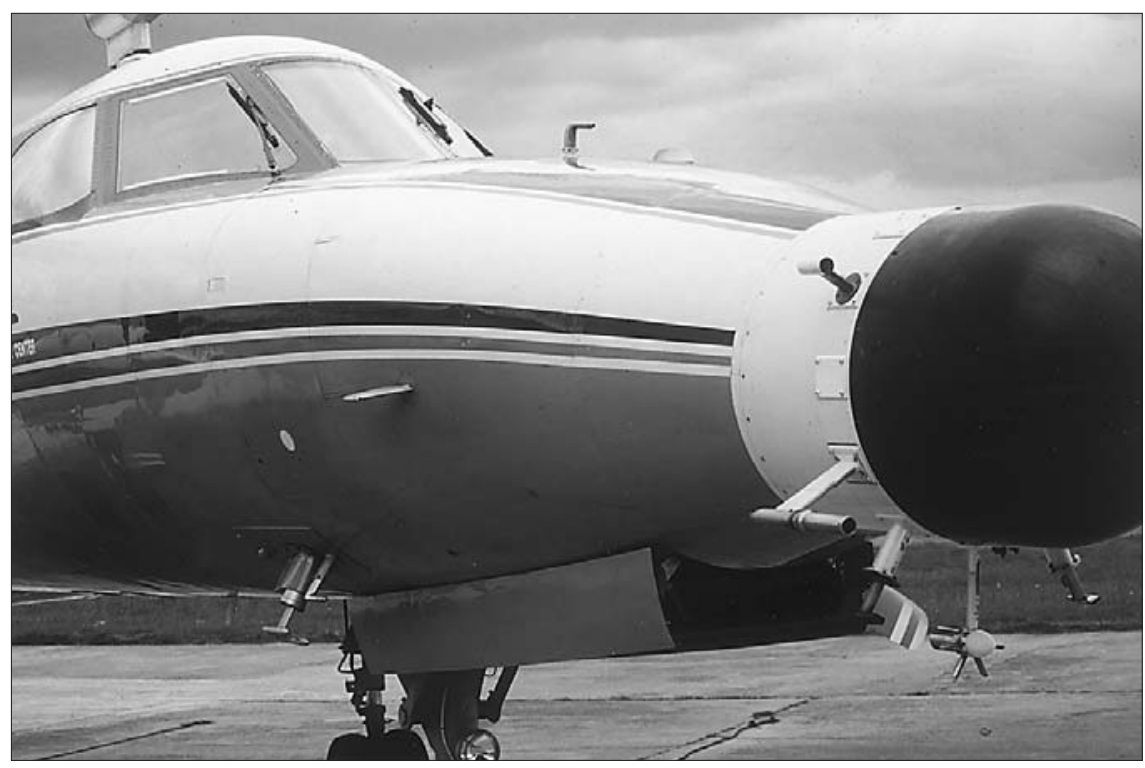

Le Piper Aztec est un bimoteur à ailes basses, non pressurisé. Il n'est pas autorisé à voler en conditions givrantes. Son autonomie de vol est de 4 heures et son plafond opérationnel de $4500 \mathrm{~m}$. Il est équipé de manière standard pour la mesure des paramètres météorologiques incluant la température, le point de rosée, la pression et le vent horizontal. Il est aussi utilisé en version télédétection avec l'instrument Polder mis en œuvre par le LOA. Il est équipé d'un système de prélèvement d'air (bouteilles en acier inoxydable) pour l'analyse en temps différé et peut effectuer la mesure des concentrations de l'ozone et des oxydes d'azote.

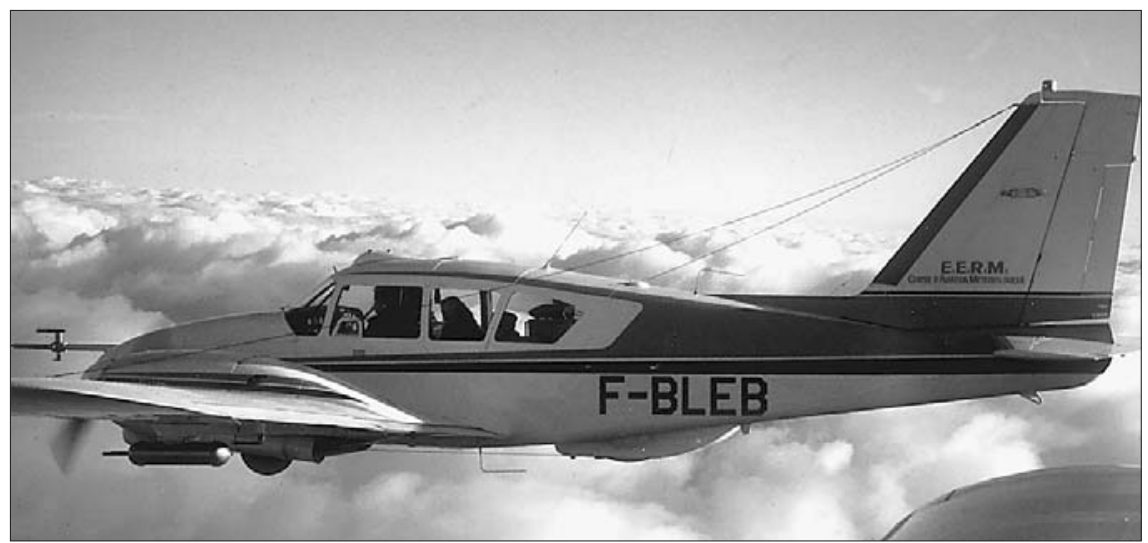

L'avion Piper Aztec survole une couche de stratocumulus. (Photo Météo-France, CNRM/CAM)

À la demande des utilisateurs, d'autres instruments (par exemple le spectroimageur Casi) peuvent être installés à bord de ces trois avions, sous réserve de compatibilité avec les possibilités d'emport et avec le reste de l'instrumentation embarquée.

L'acquisition, le contrôle, le traitement et la visualisation des données

Les trois avions sont équipés d'ordinateurs de contrôle et de systèmes d'enregistrement et de visualisation des données. Ces systèmes permettent d'acquérir plus de 150 paramètres analogiques, numériques ou d'état, à des cadences variant de 1 à $200 \mathrm{~Hz}$, choisies pour des raisons physiques ou techniques. Les données sont disponibles en temps réel sur des consoles graphiques pour permettre la conduite interactive des vols et sur support magnétique pour le traitement en temps différé.

Au sol, les avions disposent de segments informatiques mobiles qui assurent une fonction de contrôle de la qualité des données acquises et leur traitement rapide (« quick-look») afin d'affiner ou de modifier la stratégie expérimentale. Actuellement, on associe à ces traitements une fonction d'analyse des données 


\section{La mesure aéroportée et la nécessité d'une acquisition rapide}

Prenons l'exemple de la mesure de température. Supposons que l'on s'intéresse à des hétérogénéités horizontales de ce paramètre d'une longueur caractéristique d'une trentaine de mètres (cas d'une couche limite convective, par exemple).

Pour une mesure en un point fixe (mesure sur tour, sur ballon captif ou au sol), en supposant que le vent soit de $2 \mathrm{~m} / \mathrm{s}$, on observera des fluctuations de température de durée caractéristique $15 \mathrm{~s}$. Dans ce cas, un échantillonnage à $0,3 \mathrm{~Hz}$ (5 points sur la période) sera amplement suffisant pour décrire correctement ces fluctuations de température.

Pour une mesure aéroportée, en supposant que l'avion vole à $400 \mathrm{~km} / \mathrm{h}$ (environ $100 \mathrm{~m} / \mathrm{s}$ ), on observera des fluctuations de température de durée caractéristique $0,3 \mathrm{~s}$. On voit que, cette fois, il faut échantillonner au moins à $v=15 \mathrm{~Hz}$ pour décrire les hétérogénéités de température. La résolution horizontale correspondante sera $\Delta l=100 / v=7 m$ environ.

qui permet de disposer en fin de campagne d'une première version de données élaborées. L'aérodatathèque du Fokker 27, tout en assurant la compatibilité avec les systèmes précédemment utilisés par l'Insu, évolue vers un nouveau système qui fournira une vision globale de la chaîne allant du capteur à la grandeur physique reconstituée. Les points forts de ce système seront, en particulier, une grande transparence pour l'utilisateur scientifique ou technique et une facilité d'accès à l'ensemble des résultats validés, via une base de données Oracle et un serveur Web.

L'intérieur de l'Arat. Ingénieur navigant face à la palette de commande et de contrôle de l'instrumentation aéroportée dite " de l'avion de base» et concernant environ 150 paramètres. (Photo Météo-France, P. Taburet)

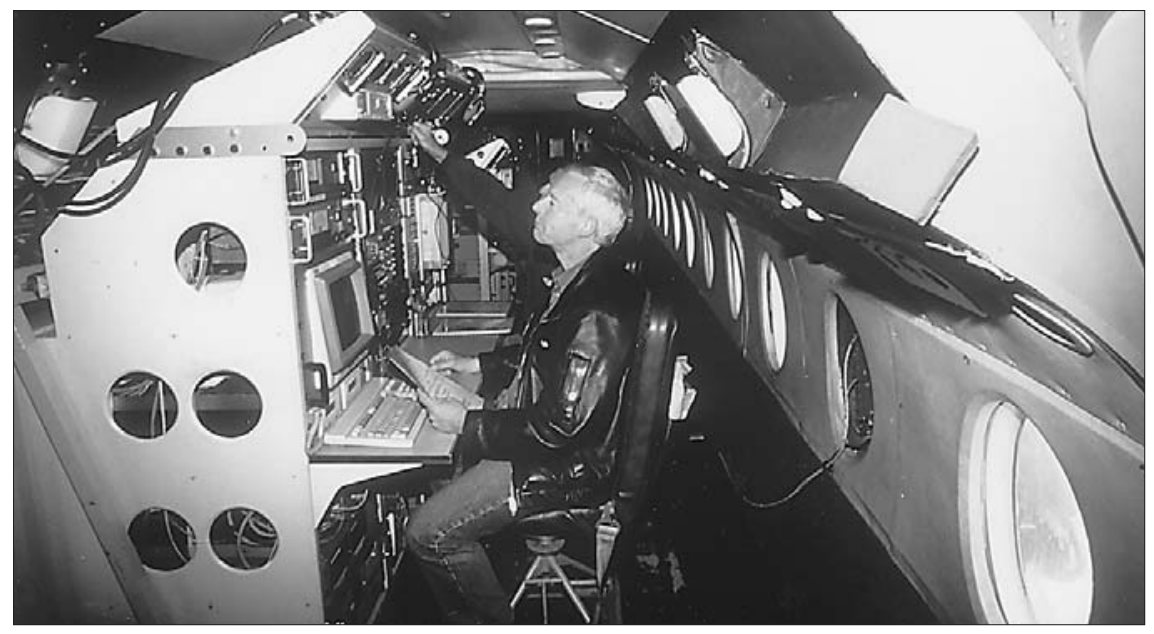

L'intérieur du Merlin IV. À gauche, au premier plan, l'ingénieur d'essai supervise le fonctionnement de l'instrumentation.

À droite, le scientifique visionne les données du radar aéroporté Ressac. (Photo Météo-France,

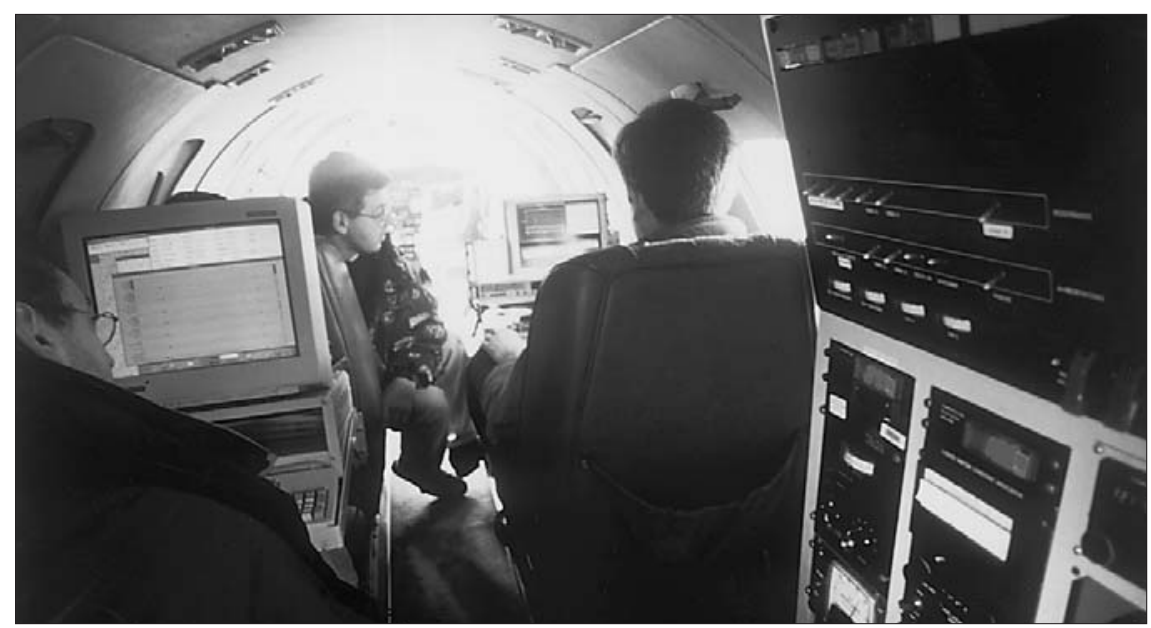


La filière Aida, développée au CNRM, permet d'assurer le traitement final des données collectées en suivant les besoins exprimés par les utilisateurs. Elle comporte une bibliothèque internationale de logiciels (Slaps) qui a été créée pour regrouper les applications sophistiquées mises au point par les experts dans l'analyse et l'interprétation des mesures aéroportées. Des interfaces conviviales ont été développées pour permettre aux utilisateurs même inexpérimentés de manipuler les données d'avion et d'en faire une analyse approfondie. Sur demande, la filière Aida peut être installée sur le site d'expérience pour une analyse en temps réel.

Dans ces évolutions, les différents systèmes tendent vers un maximum de facilité d'accès et d'homogénéité pour les utilisateurs.

\section{Décomposition d'une fonction atmosphérique en une composante lente (ou dérive) et une composante rapide (ou turbulente)}

Une fonction atmosphérique $X(p)$ définie de manière chronologique $(p=t)-c^{\prime}$ est le cas de l'observation en un point fixe - ou définie par rapport à un paramètre de position $(p=x)$ - c'est le cas de la mesure aéroportée - se présente comme une superposition de signaux recouvrant une gamme très large de fréquences ou de nombres d'ondes (figure a). On admet généralement, et cela est commode, que cette fonction peut être décrite par deux domaines spectraux disjoints :

- l'allure générale assimilée à une fonction continue et dérivable et qui représente la dérive temporelle ou géographique de la fonction (figure b) ;

- le bruit superposé qui représente la composante turbulente induite par l'agitation tridimensionnelle de l'écoulement dans des conditions de turbulence dynamique et thermique (figure $\mathrm{c}$ ).

Par définition, ce domaine turbulent est décrit par une large étendue d'échelles de mouvement, depuis les grandes échelles de la production jusqu'aux petites échelles de la dissipation. Cette étendue d'échelles donne à cette composante turbulente une structure fractale (fonction non dérivable) : un zoom de la fonction donne une fonction présentant les mêmes caractéristiques (invariance d'échelle), avec en général une énergie qui décroît lorsqu'on grossit le zoom (figure d).

En corrélant les composantes turbulentes d'une variable thermodynamique ou scalaire (concentration d'un constituant, par exemple) et de la vitesse de l'écoulement, on obtiendra une composante dite turbulente du transport de la variable (voir encadré page 25).

La fonction $\langle X\rangle$ décrivant le premier domaine sera appelée dérive, variable lente, composante lente ou signal lent. La fonction $X^{\prime}$ décrivant le bruit sera appelée fluctuation ou composante turbulente. C'est cette dernière composante qui nous intéresse pour décrire les caractéristiques statistiques du champ turbulent et pour calculer les transports qui en résultent. Pour une telle utilisation, cette composante doit être stationnaire, ce qui veut dire que ses propriétés statistiques sont indépendantes du domaine de la fonction sur lequel elles sont calculées.

Décomposition d'un signal géophysique (a) en sa dérive (b)

et sa composante turbulente (c).

Le zoom (d) illustre

l'invariance d'échelle des fonctions turbulentes.

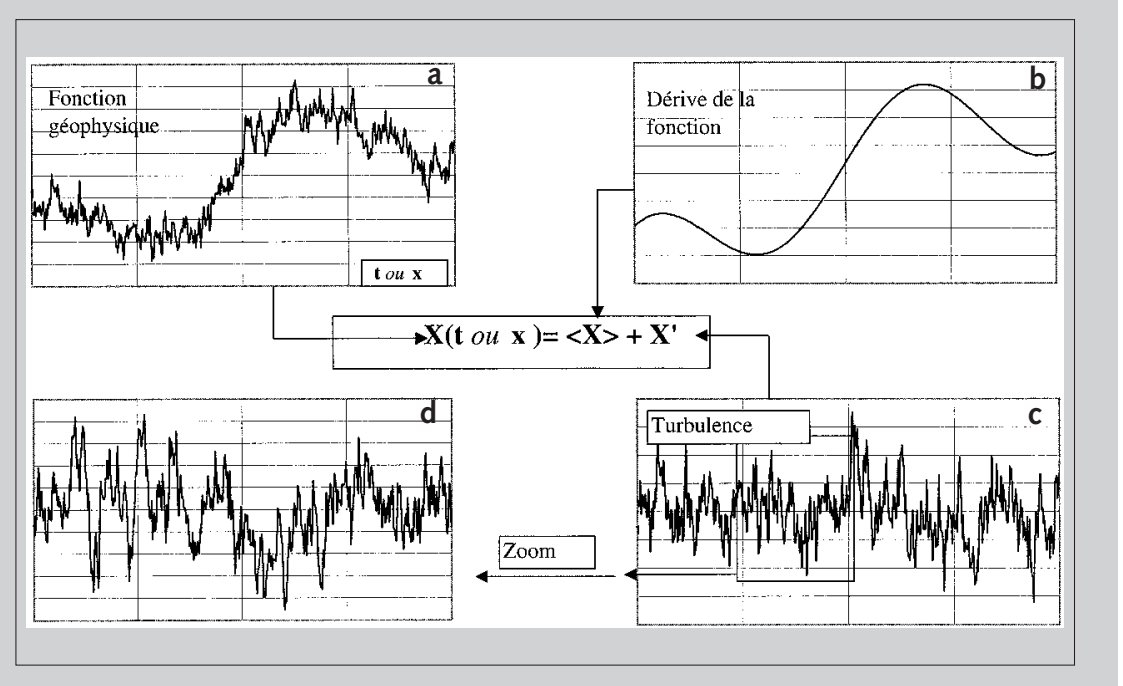




\section{Étalonnages et comparaisons}

La mesure aéroportée est une mesure complexe, dans laquelle les variables recherchées ne peuvent généralement être obtenues qu'à travers la combinaison de toute une série de paramètres dont la mesure est fortement perturbée par les conditions ambiantes (modification et perturbation des écoulements, vibrations mécaniques, perturbations électriques, mouvements de l'avion, etc.). À titre d'exemple, la mesure de la vitesse de l'air nécessite l'acquisition d'un minimum de 11 paramètres (pressions totale et statique, température, déplacement de l'avion, angles d'incidence, roulis, tangage, etc.) disponibles à des fréquences parfois différentes, allant généralement de la dizaine à la centaine de hertz. Les caractéristiques de l'avion influençant la mesure au moins autant que le capteur, la physique de la mesure et les étalonnages doivent être déterminés pour le couple avion-capteurs installés. Le choix de la position optimale des capteurs nécessite souvent l'utilisation préalable de modèles de simulation numérique ou d'essais en soufflerie. Une bonne connaissance de la fonction de transfert du système est extrêmement difficile et longue à obtenir. Toute modification d'un capteur ou de son emplacement, ainsi que tout retrait ou ajout d'un autre capteur, sont de nature à modifier l'aérodynamique et le comportement de l'avion. C'est pourquoi il est généralement recommandé d'éviter des modifications trop fréquentes des capteurs. Tout changement d'avion ou de capteur peut nécessiter une reprise totale des opérations d'étalonnage et de validation.

Ces particularités soulignent la nécessité d'effectuer régulièrement des comparaisons. Non seulement les étalonnages sont à réaliser pour chaque ensemble constitué par l'avion et son instrumentation, mais ils doivent aussi garantir la compatibilité des résultats obtenus par des avions travaillant simultanément. Cette configuration a été fréquemment utilisée lors de grandes expériences (ICE, Eucrex, Pyrex, Sofia-Astex, etc.) et a même nécessité la réalisation de campagnes spécifiques de comparaison et d'étalonnage mutuel, aussi bien pour les mesures dynamiques (Quante et al., 1996) que pour les mesures de référence comme la température du point de rosée (Ström et al., 1994). Ces procédures représentent aussi les seules méthodes disponibles pour effectuer des étalonnages en altitude.

\section{Statistique de la turbulence}

La caractérisation des propriétés statistiques d'une fonction turbulente se fait de façon simple si celle-ci répond au critère de stationnarité. Les moments turbulents $\mathrm{d}^{\prime}$ ordre $\mathrm{n}$ sont calculés comme des moyennes par rapport à $\mathrm{t}$ ou à $\mathrm{x}$ :

$M_{x}^{n}=\frac{1}{T} \int_{0}^{T} \prod_{i=1}^{n} x_{i} d t=\frac{1}{y} \int_{0}^{y} \prod_{i=1}^{n} x_{i} d x$

Les moments d'ordre 2, 3 et 4 d'une fonction $X$ - les plus employés - seront les moyennes de $X^{2}, X^{3}$ et $X^{4}$. La représentativité temporelle ou spatiale du moment calculé est ensuite critiquée en fonction de la qualité de la statistique faite. L'échantillonnage effectué en un point ou le long d'un transect est supposé avoir les mêmes caractéristiques que celles que l'on aurait en tout point du champ. Par suite de la stratification verticale de l'atmosphère, on préférera cependant passer d'une mesure ponctuelle ou spatiale à altitude constante à la propriété statistique moyenne dans le plan horizontal.

Si les i utilisés pour calculer le moment $M$ sont différents, on définira un moment croisé entre différentes composantes turbulentes. C'est cette méthode qui est employée pour calculer les transports (ou flux) turbulents (voir encadré page 25).

\section{ORGANISATION}

Bien que les avions soient mis en œuvre par des organismes distincts (MétéoFrance pour le Merlin IV et le Piper Aztec, l'IGN pour le Fokker 27 avec l'Insu pour son instrumentation atmosphérique et le Cnes pour son instrumentation télédétection), un bon niveau de cohérence et d'homogénéité a pu être maintenu grâce à la mise en place d'un Comité scientifique commun aux trois avions. Le rôle de ce comité est en particulier d'évaluer régulièrement les performances des avions (analyse des données, comparaisons...), de contrôler leur bonne utilisation (comptes rendus d'expérience, séminaires, ateliers...) et d'apporter un soutien aux groupes techniques (choix d'options, modifications, groupes d'experts...) et aux utilisateurs (réunions, mise à jour de documents techniques, bibliographie...). 
Un appel d'offres est diffusé chaque année à l'automne pour susciter des demandes d'utilisation de ces avions. Les réponses sont examinées par le Comité scientifique qui donne un avis sur l'intérêt scientifique et la faisabilité des expériences proposées. Cet avis permet d'établir si nécessaire un ordre de priorité dans la prise en compte des expériences et le niveau de la participation financière qui sera éventuellement accordée pour leur réalisation.

Dans le but de faciliter l'instrumentation et l'utilisation scientifique des avions, le Comité scientifique encourage et soutient également les propositions de développements instrumentaux et celles visant à comparer la qualité des mesures aéroportées avec celles d'autres instruments au sol ou embarqués, ainsi que les études facilitant la constitution de banques de logiciels. Il a été ainsi décidé de financer tout ou partie des projets de mesure rapide de température par ultrasons (LMD), du projet de réfractomètre micro-ondes (CETP) et de mesure des paramètres turbulents en version lisse du Fokker 27 (LA); on appelle version lisse une configuration de vol dans laquelle on n'utilise pas la perche située à l'avant de l'avion.

\section{COLLABORATIONS ET PROGRAMMES EUROPÉENS}

De nombreuses collaborations ont été lancées à l'échelle européenne (ateliers techniques, expériences de comparaison...). Ces échanges ont en particulier donné lieu à la mise en place du projet Staaarte (Scientific Training and Access to Aircraft for Atmospheric Research Throughout Europe) proposé par l'Institut national des sciences de l'univers (Insu-CNRS), le UK Meteorological Office (Grande-Bretagne) et le Deutsche Forschungsansalt für Luft- und Raumfahrt (DLR, Allemagne), en réponse à l'appel d'offres lancé par la Commission européenne (DG XII, Science, Research and Development) dans le cadre du programme Training and Mobility of Researchers (Activity : Access to Large-Scale Facilities).

Ces organismes se sont associés pour mettre des avions de recherche atmosphérique à la disposition des laboratoires européens qui n'ont généralement pas accès à ce type de moyens lourds, et donner ainsi un nouvel essor aux recherches européennes dans les domaines de la météorologie, de la climatologie et de l'environnement. Les trois avions qui ont été retenus pour leurs spécificités sont le Fokker 27 (Arat), le C130 géré par le Meteorological Research Flight (UK Meteorological Office) et le Falcon 20 géré par le DLR.

Le projet est actuellement financé par la Commission européenne pour une période de trois ans commencée en 1996. Le contrat est de 2,4 MECU. Sur ces crédits, 400000 ECU sont affectés à l'utilisation du Fokker 27 et permettront d'offrir environ 25 heures de vol par an aux équipes étrangères. Un premier appel d'offres a été diffusé en février 1996 et les diverses propositions ont été examinées par un comité d'allocation. Il faut noter que le Fokker 27, grâce à une importante flexibilité et à une grande souplesse d'emploi, a été très demandé. La première campagne Staaarte a eu lieu en septembre 1996 dans la région d'Athènes, en collaboration avec quatre laboratoires grecs, avec pour objectif de mesurer les sources de pollution atmosphérique. Le Fokker 27 était équipé du lidar ozone Alto du Service d'aéronomie (SA) et de l'analyseur d'oxydes d'azote du Laboratoire interuniversitaire des systèmes atmosphériques (Lisa). Le deuxième appel d'offres, diffusé en janvier 1998, est en cours de réalisation.

Jusqu'en 1989, les données des avions ont surtout été utilisées par des spécialistes en mesure aéroportée. À partir de 1990, avec l'extension des facilités offertes par les systèmes de vérification, de dépouillement et d'analyse des données développés par la division technique de l'Insu (Insu/DT) et le CNRM, les mesures sont devenues plus aisément accessibles aux usagers non spécialisés. Les avions ont alors joué un rôle croissant dans les grandes campagnes scientifiques (tableau 1) : le nombre de documents techniques et d'articles scientifiques reposant sur les résultats obtenus à partir des mesures de nos avions de recherche a été multiplié par 3 en quelques années. Cet article ne prétend pas décrire de façon exhaustive toutes les possibilités offertes par les moyens de mesure aéroportés, ni présenter l'ensemble des résultats obtenus, mais souhaite plutôt fournir quelques exemples illustrés à travers des applications récentes. 


\begin{tabular}{|c|c|c|c|}
\hline Expérience & Date & Lieu & Objectif \\
\hline Fetch & 1998 & Montpellier & Étude des échanges océan-atmosphère dans les cas de tramontane et de mistral \\
\hline Pollution urbaine & 1997 & Paris & Étude des épisodes de pollution en région parisienne \\
\hline ACE 2 & 1997 & Canaries & Étude microphysique des nuages en couches étendues \\
\hline Lisex & 1997 & Lisbonne & Étude de pollution (Staaarte) \\
\hline Bema & 1997 & Valencia & Étude de physico-chimie dans la couche limite \\
\hline Alpilles & 1997 & Avignon & Étude de couvert végétal \\
\hline Toast C & 1996-1997 & $\begin{array}{l}\text { Méditerranée - } \\
\text { Nord Atlantique }\end{array}$ & Étude des échanges tropostratosphériques d'ozone \\
\hline Expresso & 1996 & Bangui (RCA) & Étude physico-chimique de la couche limite (savane et forêt) \\
\hline Grèce & 1996 & Athènes & Étude de transfert de la pollution (Staaarte) \\
\hline Sesame & 1995 & Kiruna (Suède) & Étude des nuages stratosphériques polaires (PSC en anglais) \\
\hline SCMS & 1995 & Floride (États-Unis) & Étude des petits nuages convectifs \\
\hline Eucrex & 1994 & Brest & Étude des nuages \\
\hline Semaphore & 1993 & Açores & Étude de l'interface océan-atmosphère \\
\hline Trac 93 & 1993 & Beauce & Étude de la convection en plaine \\
\hline Hapex-Sahel & 1992 & Niamey (Niger) & Étude du cycle de l'eau en région semi-aride \\
\hline Sofia-Astex & 1992 & Açores & Étude de l'interface océan-atmosphère \\
\hline Elsa/EASOE & 1991 & Kiruna (Suède) & Étude des nuages stratosphériques polaires (PSC en anglais) \\
\hline Rene & 1991 & Trondheim (Norvège) & Étude de la houle et des vents en surface de l'océan (Validation ERS 1) \\
\hline Swade & 1991 & Wallops (États-Unis) & Étude de la houle et des vents en surface de l'océan \\
\hline Nuac & 1989-1991 & France & Étude des composés chimiques des précipitations \\
\hline Pyrex & 1990 & Pyrénées & Étude de l'influence des Pyrénées sur l'écoulement atmosphérique \\
\hline ICE & 1989 & Nordoltz (Allemagne) & Étude des nuages de glace \\
\hline Fronts 87 & 1987 & Brest & Étude des systèmes frontaux sur le proche Atlantique \\
\hline Toscane & 1985-1987 & Quimper & Étude de l'interface océan-atmosphère \\
\hline Hapex-Mobilhy & 1986 & Toulouse & Étude du cycle de l'eau en région tempérée \\
\hline Autan & 1984 & Toulouse & Étude des vents locaux \\
\hline Landes-Fronts 84 & 1984 & $\begin{array}{l}\text { Sud-Ouest } \\
\text { de la France }\end{array}$ & Étude des systèmes frontaux convectifs \\
\hline Nephos & 1982 & Lannion & Étude des caractéristiques des nuages de grandes dimensions \\
\hline Alpex & 1982 & Fréjus & Étude des écoulements autour des Alpes \\
\hline PEP & 1980-1981 & Valladolid (Espagne) & Étude microphysique des nuages \\
\hline
\end{tabular}

Tableau 1 - Principales campagnes expérimentales réalisées avec les avions français de recherche atmosphérique et de télédétection.

\section{Variation spatiale de l'indice de réfraction}

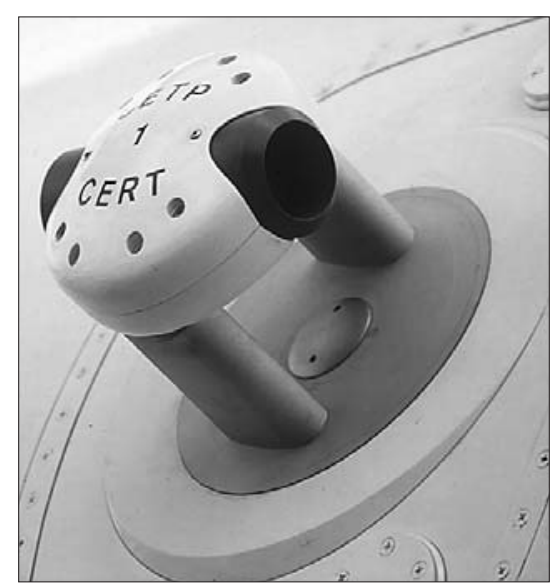

Vue extérieure du Merlin IV montrant le réfractomètre micro-ondes aéroporté, monté sur un hublot de l'avion. (Photo Météo-France, P. Taburet)
Les champs d'indice de réfraction de l'atmosphère ont une influence importante sur la propagation des ondes électromagnétiques et sur les liaisons radioélectriques. Un des objectifs de l'expérience Trac 93 (Campistron et al., 1995) était l'étude de la rétrodiffusion en air clair des ondes électromagnétiques en bande C. Au cours de cette expérience, le radar Doppler Ronsard $(5,4 \mathrm{~cm}) \mathrm{du}$ CETP a enregistré des données de réflectivité en air clair, principalement dans la couche limite atmosphérique, jusqu'à une altitude de $2,5 \mathrm{~km}$ et sur une distance horizontale d'environ $25 \mathrm{~km}$. Conjointement, les fluctuations de l'indice de réfraction étaient mesurées avec le réfractomètre micro-ondes $10 \mathrm{GHz}$ du CETP (Delahaye et Lavergnat, 1994) qui équipe l'avion. Les mesures ont mis en évidence des régions où la rétrodiffusion des ondes radar est bien supérieure à celle qui aurait pu être déduite de la valeur du «coefficient de structure » de l'indice de réfraction (connu par les spécialistes sous le nom de $\mathrm{Cn}^{2}$ ). Pour expliquer cette différence, Lohou et al. (1995) ont proposé une hypothèse fondée sur la présence, dans la structure turbulente, de fortes discontinuités de l'indice de réfraction. Ces zones de transition sont en désaccord avec la loi d'invariance d'échelle sur laquelle repose la relation réflectivité-coefficient de structure de l'indice (formule de Tatarskii). Les mesures ont montré que les discontinuités de très petite échelle sont des sources très efficaces de rétrodiffusion des ondes électromagnétiques. Elles ont permis de mettre en évidence, à l'échelle de la demi-longueur d'onde du radar, des variations locales de l'indice de réfraction 200 fois supérieures à ce que laisserait prévoir une fonction turbulente respectant l'invariance d'échelle et utilisant le même coefficient de structure (figure 1). 


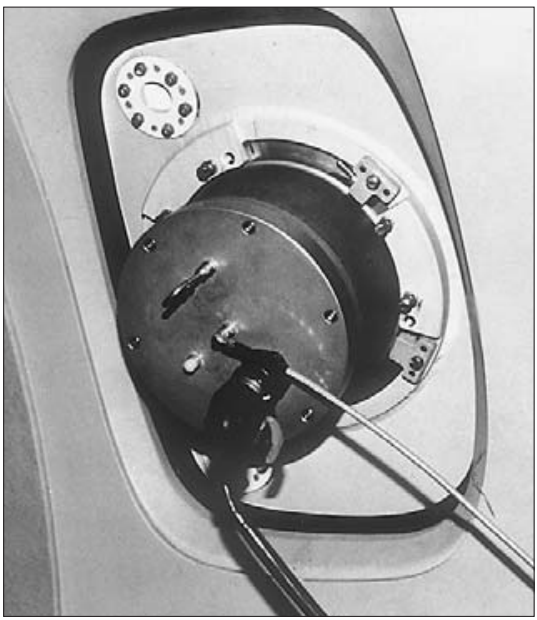

Vue intérieure du Merlin IV montrant l'électronique du réfractomètre micro-ondes aéroporté. (Photo Météo-France, P. Taburet)
Une autre retombée de cette étude a été la mise en évidence des possibilités de la réfractométrie pour la mesure du flux de chaleur latente (ou de vapeur d'eau). Classiquement, cette mesure est faite par mise en œuvre de capteurs fonctionnant sur le principe de l'absorption optique dans le domaine de l'infrarouge ou de l'ultraviolet. Ces capteurs effectuent le plus souvent des mesures relatives et nécessitent des étalonnages permanents. L'indice de réfraction de l'air dépend, par ordre d'importance, de la pression partielle de vapeur d'eau, de la température et de la pression. Dans la couche limite atmosphérique, les fluctuations de la pression partielle de vapeur d'eau ont une contribution prédominante, sauf près de la surface où la contribution des fluctuations de température doit aussi être prise en compte. Le réfractomètre qui équipe le Merlin IV permet de faire des mesures absolues avec une résolution supérieure au 1/30 de seconde. Dès lors, il est parfaitement adapté à la mesure absolue du flux de chaleur latente. Pour illustrer cette application, la figure 2 présente une comparaison des covariances entre vitesse verticale w et rapport de mélange de la vapeur d'eau q (covariances utilisées pour l'évaluation du flux de vapeur d'eau, voir l'encadré ciaprès) respectivement obtenues à partir des données du réfractomètre et de celles d'un capteur à absorption dans l'ultraviolet (raie Lyman alpha). Une part de la variabilité observée est associée à l'étalonnage de l'appareil à absorption Lyman alpha (Gervaise et Loubet, 1994).

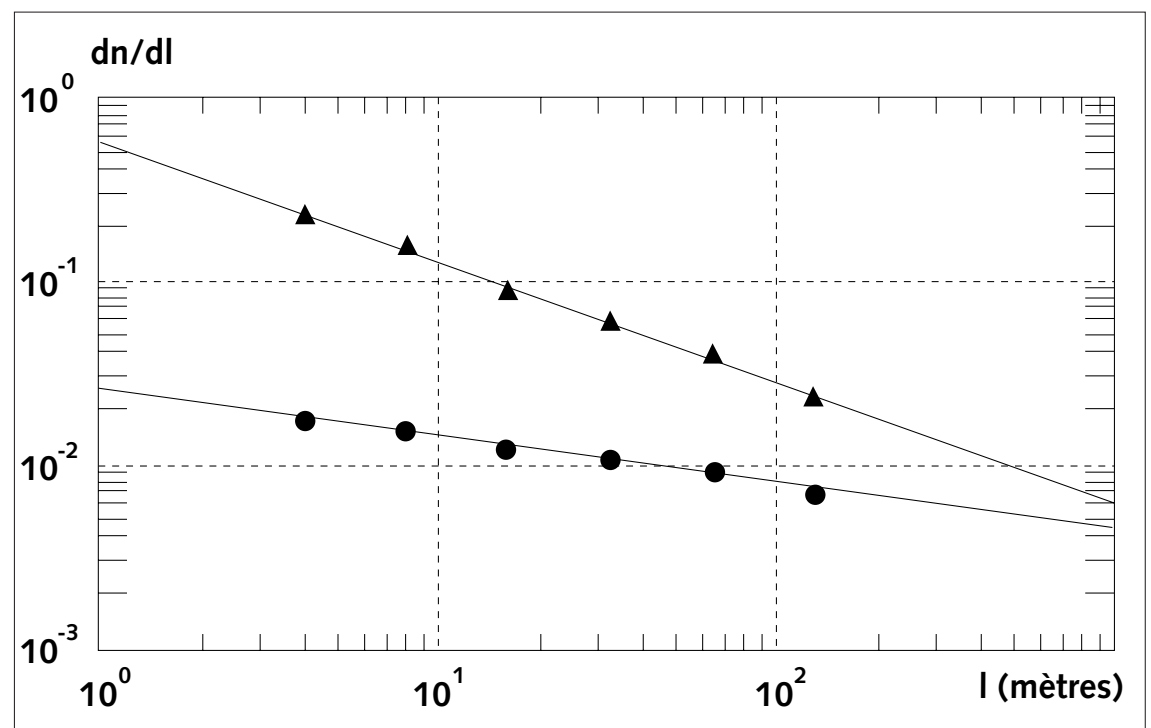

Figure 1 - Variation comparée de la dérivée spatiale de l'indice de réfraction de l'air $\mathrm{dn} / \mathrm{dl}$ (en unités d'indice par mètre). Les deux fonctions comparées ont la même valeur de coefficient de structure $\mathrm{Cn}^{2}$. Les cercles correspondent à l'évolution de la dérivée pour une fonction répondant strictement au critère d'invariance d'échelle (forme canonique à domaine inertiel en -5/3). Les triangles correspondent à une fonction contenant des discontinuités comme les fonctions indices mesurées. Le calcul est limité à la bande spectrale de mesure en avion (valeur minimale de I de l'ordre de $4 \mathrm{~m})$. À la demi-longueur d'onde du radar $(2,7 \mathrm{~cm}$, hors figure), la dérivée de la fonction contenant des discontinuités d'indice est 200 fois plus grande que la dérivée de la fonction canonique. (D'après Lohou et al., 1995)
Figure 2 - Mesure du flux d'évaporation à l'aide du réfractomètre $10 \mathrm{GHz}$ du CETP. La covariance w'q' calculée à partir de la fluctuation absolue de l'indice de réfraction est comparée à la covariance $w^{\prime}$ q' calculée à partir de la fluctuation relative issue de la mesure par absorption UV (Lyman alpha) et étalonnée par rapport au point de rosée. w' est la fluctuation de la vitesse verticale et q' celle du rapport de mélange de la vapeur d'eau. (D'après Gervaise et Loubet, 1994)

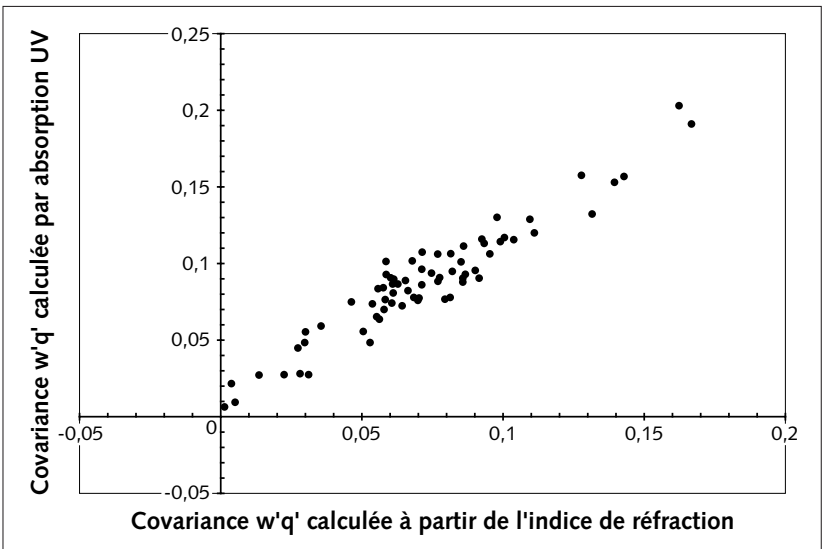




\section{Méthode de corrélation et calcul des flux turbulents}

Compte tenu des échelles horizontale et verticale de l'atmosphère, on conçoit intuitivement que le domaine dans lequel la turbulence sera la plus efficace est celui du transport vertical. Au-dessus d'une surface plane et hors système perturbé, la vitesse verticale de l'air est en moyenne nulle. L'agitation turbulente prend alors le relais pour assurer le transport de matière et d'énergie entre la surface et l'atmosphère libre. Le flux vertical turbulent de la quantité $X$ correspond à la moyenne du produit $w X$ au travers d'une surface horizontale, w étant la vitesse verticale. L'hypothèse selon laquelle la statistique en un point au cours du temps ou le long d'une trajectoire horizontale est la même que la statistique en tout point du plan horizontal prend ici toute son importance puisque l'on procédera en pratique à un changement de variable d'intégration :

$$
<w X>\overline{w X}=\frac{1}{S} \int_{S} w^{\prime} X^{\prime} d s=\frac{1}{T} \int_{0}^{T} w^{\prime} X^{\prime} d t=\frac{1}{y} \int_{0}^{y} w^{\prime} X^{\prime} d x
$$

En turbulence tridimensionnelle pleinement développée et stationnaire, cette hypothèse est parfaitement acceptable. Par contre, la source thermique de la turbulence atmosphérique induit une dissymétrie entre les trois composantes du transport, puisque la flottabilité liée à la densité est portée par la verticale. On sait par ailleurs que l'instabilité thermique et dynamique peut donner naissance à des " circulations cohérentes » qui mettent en défaut la représentativité de la statistique unidimensionnelle (1D).

Admettons que les statistiques $1 \mathrm{D}$ et $2 \mathrm{D}$ soient identiques dans le plan horizontal étudié. La moyenne $\langle w X\rangle$ correspond alors au transport vertical turbulent de $X$ au travers de ce plan. Sa valeur dépend de l'intensité de l'agitation de $w$ et de $X$, mais surtout de leur corrélation. Si cette corrélation était nulle, il ne résulterait de l'agitation de la vitesse verticale et de la quantité $X$ aucun transport vertical. Dans tous les autres cas, il y a transport vertical turbulent vers le haut ou vers le bas suivant le signe positif ou négatif de la corrélation $\langle w X\rangle$.

\section{Étude des flux de quantité de mouvement}

Les données in situ obtenues à partir de plusieurs avions volant de façon coordonnée permettent la caractérisation des écoulements atmosphériques dans une gamme étendue d'échelles. Une illustration de ces capacités peut être présentée à travers certains résultats de l'expérience Pyrex. Les études (Bougeault et al., 1996) portaient sur les mécanismes de franchissement et de contournement par l'air du massif pyrénéen et sur la formation des vents locaux (tramontane sur la Méditerranée, autan dans le Lauraguais, cierzo dans la vallée de l'Èbre). De nombreuses échelles ont été abordées. L'étude des mécanismes de franchissement a été effectuée grâce à un transect de $200 \mathrm{~km}$ de long, de $12 \mathrm{~km}$ de haut et perpendiculaire à la chaîne de montagne. Les mesures des trois avions français étaient complétées en altitude (entre 6 et $12 \mathrm{~km}$ ) par celles de l'avion Falcon 20 du DLR. Les champs obtenus grâce aux mesures coordonnées ont permis d'effectuer un bilan de la quantité de mouvement et une comparaison avec les résultats des modèles hydrostatiques Peridot et Salsa. Les mesures par avion associées à celles des ballons plafonnants ont permis d'analyser les ondes de sillage. Ces résultats ont ensuite servi à valider les modèles non hydrostatiques et à ajuster la paramétrisation dans les modèles opérationnels.

Les mesures des avions ont également permis de caractériser la turbulence de petite échelle résultant des zones de cisaillement et de dégénérescence des ondes. Une étude spécifique de la turbulence de sillage a pu être réalisée, grâce aux vols effectués au voisinage du relief et au développement d'outils nouveaux de type ondelettes. La structure des fonctions turbulentes mesurées est caractérisée par une forte « instationnarité ». C'est le cas en particulier pour la turbulence observée dans les vents locaux, mais aussi et surtout dans le sillage du relief (Attié, 1994 ; Attié et al., 1997). La figure 3 présente une restitution de la vitesse verticale et fait apparaître une superposition complexe de mécanismes d'échelles bien différentes comportant en particulier un mélange d'ondes de sillage et de turbulence. Pour traiter ce type de fonction de structure, des outils nouveaux sont développés ou adaptés : les méthodes conditionnelles et les méthodes reposant sur la transformée en ondelettes (Druilhet et al., 1994). La figure 4 illustre la 
Figure 3 - Coupes horizontales de la vitesse verticale de l'air dans un plan perpendiculaire à la chaîne des Pyrénées (transect Pyrex) pour trois altitudes comprises entre 4 et $6 \mathrm{~km}$. En amont de la chaîne les vitesses verticales sont très faibles. Le franchissement se caractérise par un mouvement ondulatoire dont la longueur d'onde est de l'ordre de $10 \mathrm{~km}$ et l'amplitude de $5 \mathrm{~m} / \mathrm{s}$. Après la zone ondulatoire, sous le vent, se trouve une zone de turbulence.

méthode d'analyse par ondelettes appliquée à une fonction instationnaire. Ce type d'analyse se substitue à l'approche par transformée de Fourier habituellement appliquée à la caractérisation spectrale des fonctions turbulentes stationnaires : elle permet une représentation temps-fréquence ou espace-nombre d'ondes et la localisation des processus énergétiques. De ce fait, ces derniers peuvent être localisés dans le temps ou dans l'espace, tandis qu'une information d'échelle caractéristique des processus est fournie par la représentation dans l'espace des phases. Dans le cas présenté ici, l'amplitude du signal transmis par le filtre est représentée. Cette méthode permet une analyse de phase, de hiérarchisation ou de corrélation entre différentes fonctions.

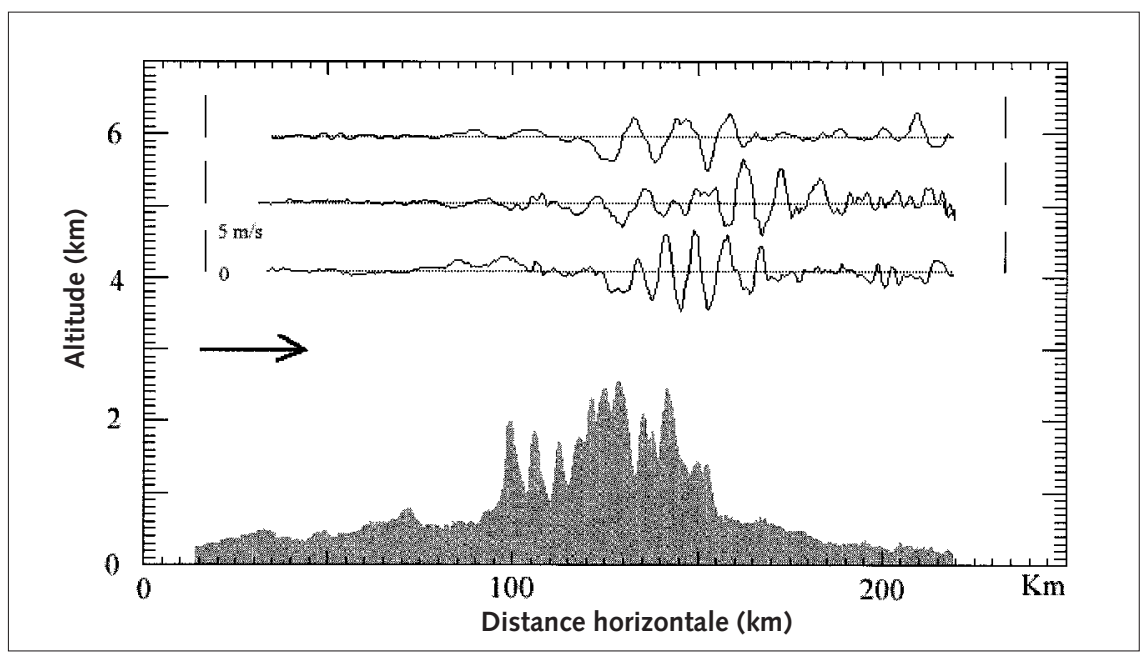

Plus récemment, les avions français ont participé à de grands programmes d'étude des interactions surface-atmosphère. Des progrès ont été réalisés sur la paramétrisation des flux de surface entre l'océan et l'atmosphère à partir de mesures comparées entre avions et bateaux (campagnes Sofia-Astex en 1992 et Semaphore en 1993). L'interaction atmosphère-surface continentale a été intensivement étudiée au cours de l'expérience Hapex-Sahel, qui s'est déroulée au Niger en 1992. Son objectif était l'étude des paramètres déterminant le bilan d'énergie et le bilan hydrique représentatifs de l'écosystème sahélien à l'échelle d'un degré carré, c'est-à-dire d'une surface comparable à celle de la maille des modèles atmosphériques.

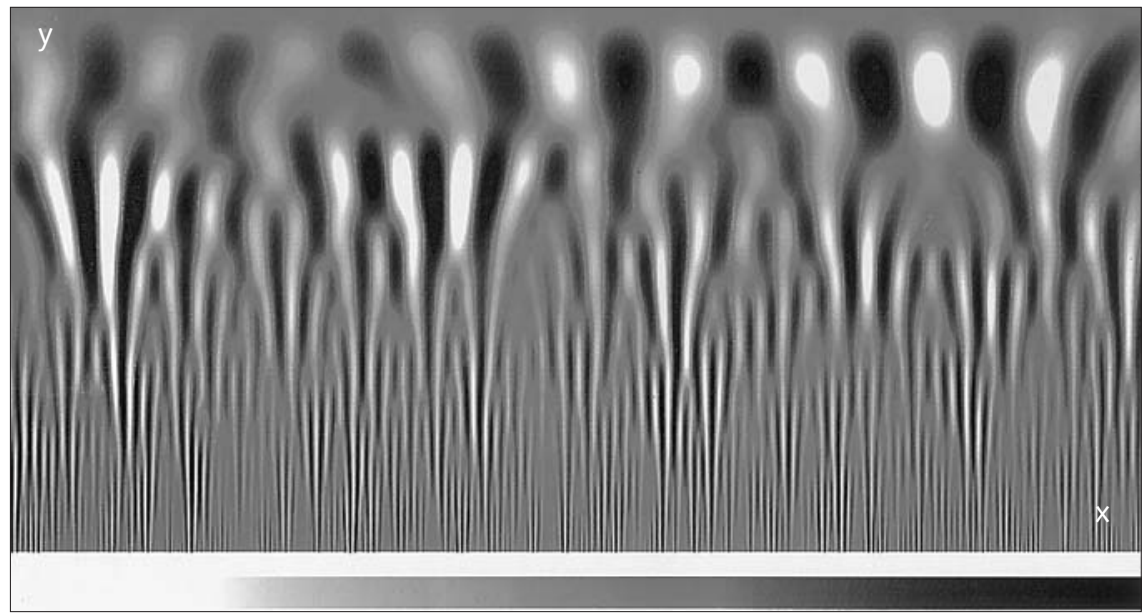

Figure 4 - Exemple d'analyse temps-fréquence d'une fonction « instationnaire ». Ce type d'analyse se substitue à l'approche par transformée de Fourier habituellement appliquée à la caractérisation spectrale des fonctions turbulentes stationnaires. La coordonnée $Y$ représente le logarithme de la fréquence ou du nombre d'ondes. La coordonnée $X$ représente la position dans le temps ou suivant une coordonnée d'espace. Le niveau de gris est proportionnel à l'amplitude du signal fourni par le filtre appliqué. Cette analyse permet de localiser les zones énergétiques de turbulence par rapport aux deux coordonnées utilisées. (D'après Druilhet et al., 1994) 
Un des objectifs de l'expérience Trac 93 précédemment citée était la recherche de mouvements cohérents développés au sein de la couche limite atmosphérique. L'organisation de tels systèmes est pressentie depuis fort longtemps. Ils sont souvent tracés par la structure nuageuse (rues de cumulus) et utilisés par les vélivoles. Du point de vue du chercheur, ce problème est extrêmement attrayant : comment l'organisation naît-elle du « chaos » turbulent ? Comment se fait le transfert d'énergie entre les petites échelles de production de la turbulence, l'échelle des courants convectifs cohérents et le passage vers la dissipation ? Les conséquences pour les modèles opérationnels de prévision du temps ne sont pas moins importantes : comment se gère le bilan d'énergie cinétique turbulente dans un système cohérent ? Comment faut-il adapter les paramétrisations admises en couche turbulente pleinement développée ? Que deviennent les descriptions statistiques de la turbulence ? Ce dernier point peut conduire à une réévaluation des méthodes de mesure fondées sur cette approche statistique : la méthode des corrélations par exemple. L'expérience Trac a permis de mettre en évidence, à partir des champs de réflectivité et des champs de vitesse radiale, l'existence d'organisations cohérentes périodiques au sein de la couche limite soumise à une instabilité turbulente thermique et dynamique. La figure 5 présente un exemple du champ de réflectivité dans un plan vertical passant par le radar. Elle montre principalement l'existence de cheminées ascendantes traversant toute la couche limite dont l'épaisseur est ici de 1,5 km. Au-dessus, l'observation radar met en évidence des maxima de réflectivité qui correspondent aux cumulus alimentés par ces ascendances. Les données de l'observation sont ensuite reprises dans un modèle non hydrostatique qui permet de décrire plus complètement la circulation, de chiffrer le transport qu'elle assure, d'étudier la sensibilité aux paramètres directeurs et, par là, de généraliser les résultats.

Figure 5 - Champ de réflectivité obtenu en air clair par le radar Ronsard $(5,4 \mathrm{~cm})$ du CETP. La coupe est réalisée suivant un plan vertical passant par le radar et s'étendant horizontalement sur $50 \mathrm{~km}$ et verticalement sur $2,5 \mathrm{~km}$

L'ensemble de la couche limite convective est décrit. Elle est caractérisée

par des ascendances qui affectent toute son épaisseur. Ces ascendances sont surmontées par des cumulus qui se traduisent

malgré leur faible développement, par une augmentation des échos.

La position des cumulus est précisée grâce au flux visible incident

mesuré à partir de l'avion Merlin IV dans le plan d'observation (courbe noire). La trace du palier de l'avion est représentée par une ligne horizontale.

\section{La microphysique des nuages chauds}

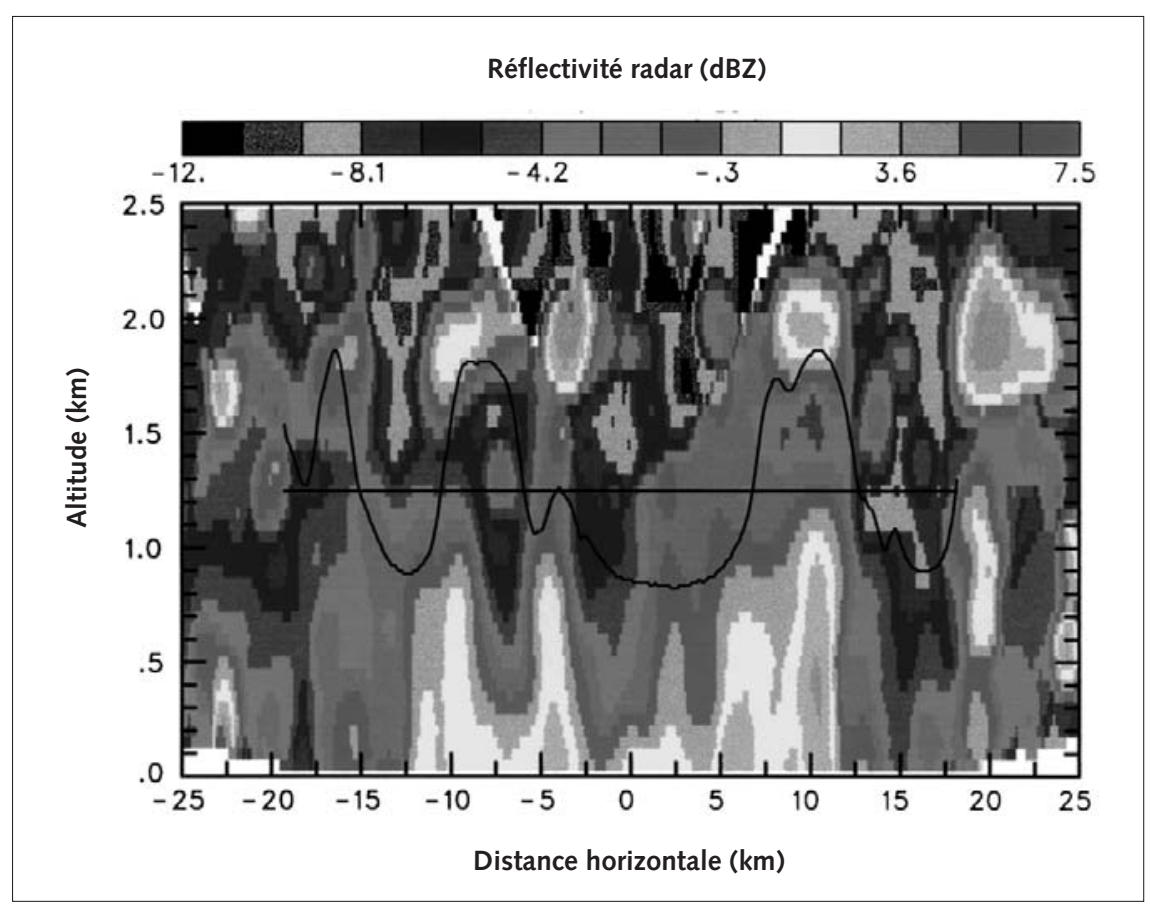

La microphysique des nuages chauds se rapporte aux processus de formation des gouttelettes de nuage, à leur croissance (processus de condensation), à leur interaction avec le rayonnement (propriétés radiatives des nuages) et à la formation des précipitations. Le paramètre essentiel pour ces études est la distribution dimensionnelle - ou spectre - des gouttelettes.

La mesure des spectres est effectuée à partir des avions de recherche à l'aide d'un granulomètre à diffusion laser : le Forward Scattering Spectrometer Probe (FSSP) de Particle Measuring Systems (PMS). Les particules qui traversent le volume d'échantillonnage de l'instrument sont comptées une à une et leur diamètre est évalué. La distribution dimensionnelle des gouttelettes est alors obtenue en cumulant les comptages par classe de diamètre. Cependant, les sources d'incertitude associées à cette mesure sont nombreuses : certaines relèvent de 


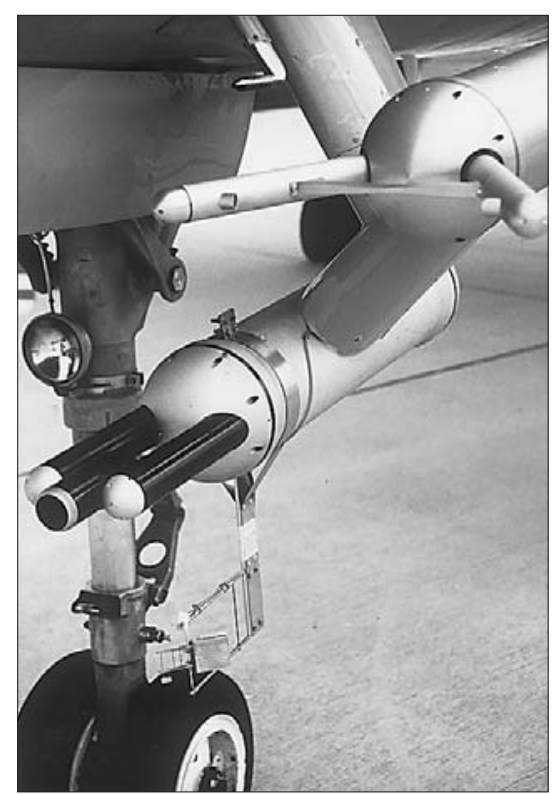

Fixés sur le Merlin IV, deux spectromètres à laser pour la mesure de la granulométrie des gouttelettes de nuage. En haut, le modèle OAP pour les grosses gouttelettes. En bas, le Fast FSSP pour la mesure rapide des petites gouttelettes. (Photo Météo-France, CNRM/CAM)

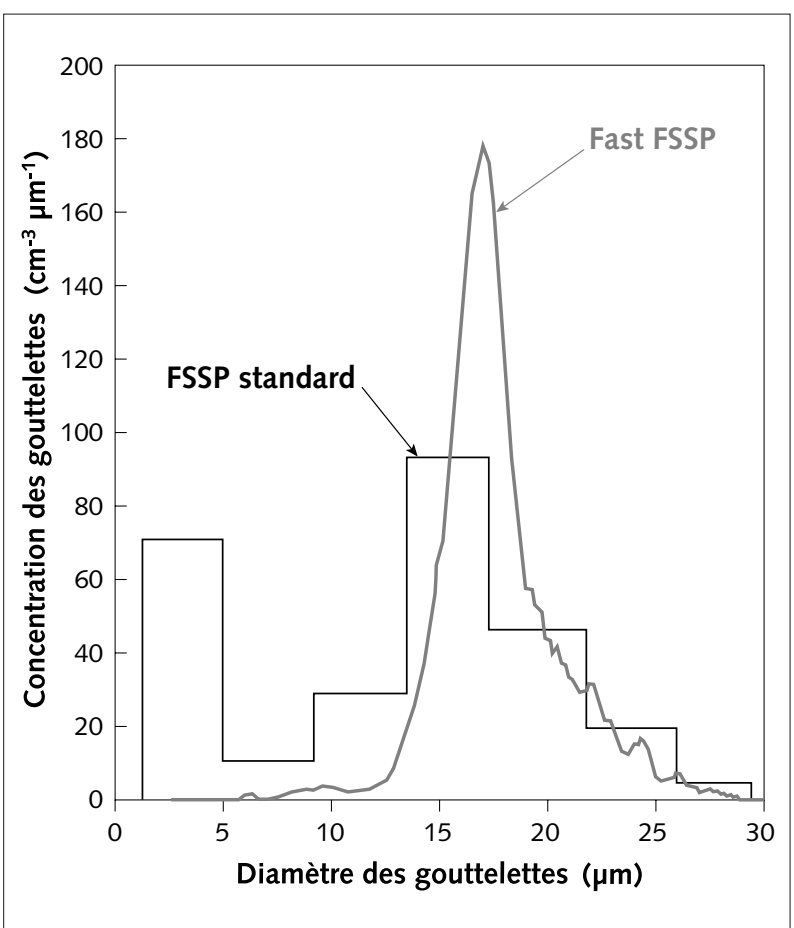

Figure 6 - Comparaison entre les mesures de la distribution dimensionnelle des gouttelettes dans un cumulus obtenues avec un FSSP standard et avec le Fast FSSP. l'instrument, d'autres sont inhérentes au principe de la mesure. Pour les premières, il s'agit d'erreurs principalement dues à la présence simultanée de plusieurs particules dans le faisceau de détection (Brenguier et Amodei, 1989 ; Brenguier, 1989 ; Brenguier et al., 1993 ; Brenguier et al., 1994) et au bruit qui affecte la mesure. Pour réduire ces erreurs, le granulomètre a été modifié au CNRM, équipé d'une nouvelle optique et d'un module électronique de traitement numérique des impulsions détectées à $16 \mathrm{MHz}$ (Brenguier, 1993 ; Brenguier et al., 1998a). Une nouvelle technique d'étalonnage a été développée ; elle permet d'étalonner l'instrument directement à partir des données collectées en vol (Coelho, 1996 ; Coelho et al., 1996). La figure 6 compare les spectres de gouttelettes mesurés dans un cumulus avec le nouvel instrument nommé Fast FSSP et avec un FSSP standard. Elle montre bien la différence qui existe entre les deux mesures en termes de résolution en diamètre des gouttelettes.

La deuxième source d'erreur est propre à tout instrument de type compteur. Le comptage étant un processus aléatoire, il est nécessaire d'observer un grand nombre de particules avant d'atteindre une signification statistique satisfaisante. Ainsi, précision de la mesure et résolution spatiale sont-elles difficilement conciliables, surtout lorsque les mesures sont effectuées à partir d'un avion qui se déplace rapidement pendant la mesure. De ce fait, le cumul fournit une très mauvaise estimation du taux de comptage. Les incertitudes ont pu être réduites grâce à l'adaptation d'un estimateur optimal non linéaire pour les processus de Poisson (Pawlowska et al., 1997) qui permet, pour un niveau de signification statistique donné, d'améliorer la résolution spatiale d'un facteur 100. La figure 7 montre une comparaison entre l'évolution temporelle de la concentration des gouttelettes dans un cumulus calculée par la méthode courante des cumuls à $10 \mathrm{~Hz}$ (a) ou à $100 \mathrm{~Hz}$ (b), et celle qui a été calculée avec l'estimateur optimal à $1000 \mathrm{~Hz}$ (c). Cette figure montre qu'à partir de $100 \mathrm{~Hz}$, dans le cas d'une mesure classique, le bruit d'origine aléatoire empêche toute estimation satisfaisante des variations de la concentration, alors que l'emploi de l'estimateur optimal permet de rejeter efficacement ce bruit et de détecter des transitions rapides de la concentration. Les progrès ainsi réalisés sur la qualité de la mesure et sur son traitement ont permis d'aboutir à une vision nouvelle des processus de condensation dans les nuages et font du Fast FSSP l'instrument de référence pour ces études.

Le premier champ d'application a été l'étude de la croissance des gouttelettes de nuages pour comprendre les processus de formation des précipitations. En effet, la théorie de croissance par diffusion de vapeur prédit la formation de spectres de gouttelettes très étroits, presque monodispersés, qui sont peu efficaces pour donner naissance aux embryons de précipitations. La turbulence dans le nuage et le mélange de l'air nuageux avec l'air environnant plus sec peuvent être responsables d'un certain élargissement des spectres, mais s'accompagnent d'une dilution de la quantité d'eau liquide qui est un facteur défavorable (Brenguier, 1990 ; Brenguier, 1991 ; Brenguier et Burnet, 1996). Dans les régions non diluées d'un nuage, on n'a pas encore pu trouver de mécanisme permettant d'expliquer de tels élargissements. Les mesures avec un instrument standard montrent des spectres beaucoup plus larges que les spectres prédits. On ne peut exclure que ces élargissements soient simplement associés à des perturbations de la mesure. Le Fast FSSP a permis d'observer des spectres beaucoup plus étroits (fig. 6), bien qu'encore trop larges par rapport à ceux attendus (Brenguier et Chaumat, 1996). L'analyse de plus d'une centaine de tels spectres observés à des altitudes variées au-dessus de la base des nuages a conduit à la mise au point d'une paramétrisation de l'élargissement des spectres (Brenguier et Chaumat, 1998). Celle-ci sert de référence pour valider les schémas numériques qui tentent d'expliquer l'origine de cet élargissement.

Des mesures récentes dans les stratocumulus ont permis de valider de nouveaux schémas de paramétrisation pour les modèles de grande échelle (Descloitres et al., 1996 ; Pawlowska et Brenguier, 1996 ; Pawlowska et al., 1998). En particulier, il a été démontré que l'épaisseur optique des nuages étendus 
Figure 7 - Calcul en fonction du temps de la concentration des gouttelettes dans un nuage : comparaison entre la méthode classique et l'estimateur optimal.

En a, les comptages sont cumulés sur une période de $0,1 \mathrm{~s}(10 \mathrm{~Hz})$. En b, la même méthode est mise en œuvre avec une période de $0,01 \mathrm{~s}(100 \mathrm{~Hz})$. La très forte variabilité de la concentration à $100 \mathrm{~Hz}$ n'a pas de sens physique : elle reflète simplement

le caractère aléatoire du comptage des particules (bruit poissonien).

En c, les données ont été traitées en utilisant l'estimateur optimal avec une résolution temporelle de $1 \mathrm{~ms}(1 \mathrm{kHz})$.

L'estimateur filtre très efficacement le bruit poissonien, tout en permettant la détection des transitions rapides de la concentration.

Caractéristiques microphysiques des cirrus et des traînées de condensation était principalement déterminée par l'épaisseur du nuage et que la concentration des gouttelettes intervenait au deuxième ordre (effet indirect). De plus, ces mesures ont fourni la première démonstration de l'effet indirect à l'échelle d'un système nuageux (Brenguier et al., 1998b). Une autre application concerne l'étude des sources de réflectivité radar dans les nuages, en s'appuyant sur les performances du Fast FSSP et de l'estimateur optimal pour documenter les transitions étroites du rapport de mélange en eau liquide dans les nuages cumuliformes (Baker et al., 1998).
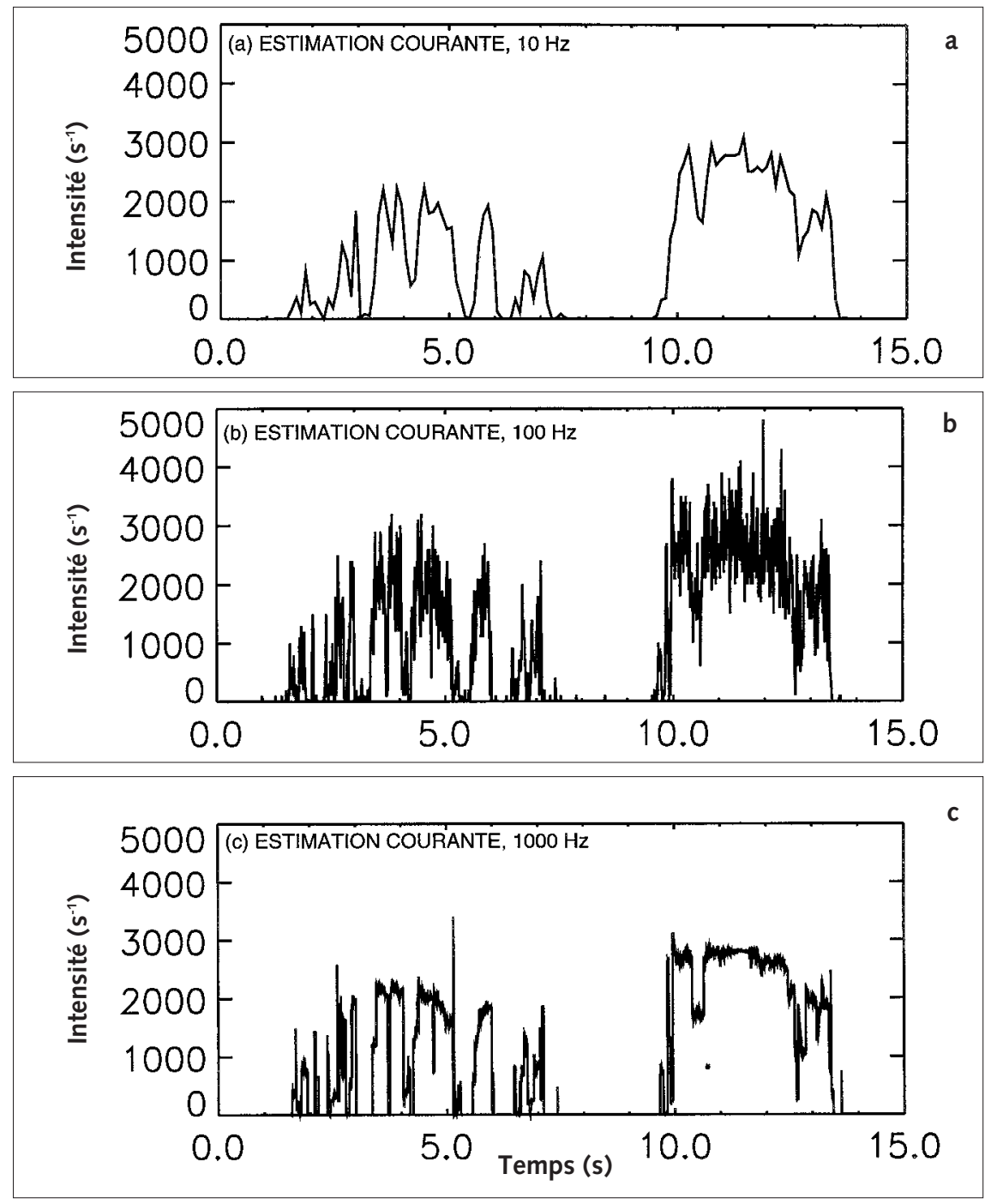

L'observation in situ des propriétés microphysiques des nuages d'altitude (cirrus et traînées de condensation dans le sillage des avions) est un des moyens essentiels pour comprendre l'impact de ces nuages sur le climat de la planète. Les programmes européens ICE (International Cirrus Experiment) et Eucrex (European Cloud and Radiation Experiment) ont été l'occasion d'échantillonner différentes situations caractérisées par des cirrus naturels et des traînées de condensation dans le sillage d'avions commerciaux.

Le traitement systématique des mesures microphysiques montre que, comparativement aux propriétés des cirrus naturels, les traînées de condensation sont caractérisées par un nombre beaucoup plus élevé de cristaux de glace (typiquement 800 par litre contre 150 par litre), mais dont le diamètre est notablement plus petit (typiquement $15 \mu \mathrm{m}$ contre $30 \mu \mathrm{m}$ ). Par ailleurs, dans ce type de nuage, l'analyse des mesures suggère que les particules de glace sont de forme sphérique (Gayet et al., 1996a). Cette hypothèse a été confirmée par l'interprétation des mesures lidar (longueur d'onde 1,02 $\mu \mathrm{m}$ ) et radiométriques infrarouges obtenues simultanément dans les mêmes champs nuageux (Febvre, 1994). En effet, la détermination subséquente du rapport $\mathrm{k}$ du coefficient de rétrodiffusion au coeffi- 


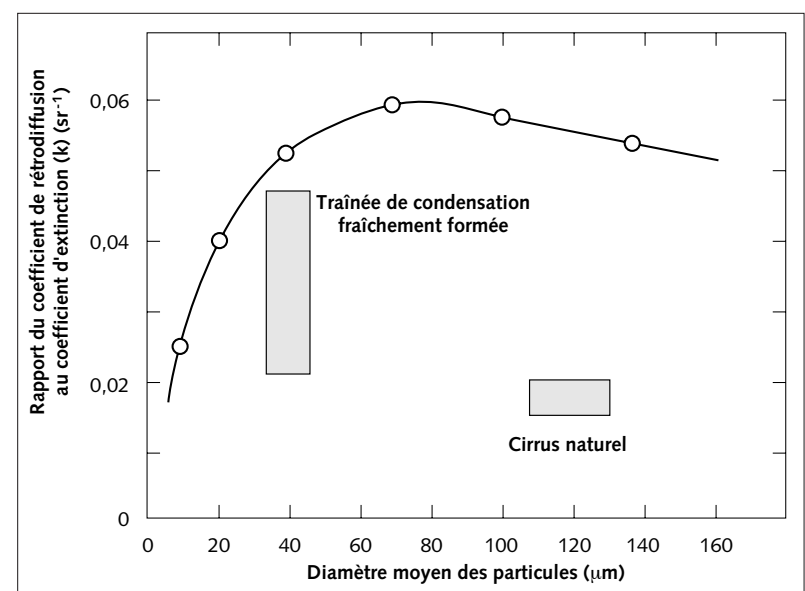

Figure 8 - Valeurs théoriques du rapport du coefficient de rétrodiffusion au coefficient d'extinction en fonction du diamètre moyen des particules (supposées sphériques). Les valeurs expérimentales déterminées au moyen de mesures in situ et télédétectées sont également reportées en fonction du type de nuage (cirrus naturel et traînées de condensation dans le sillage des avions).

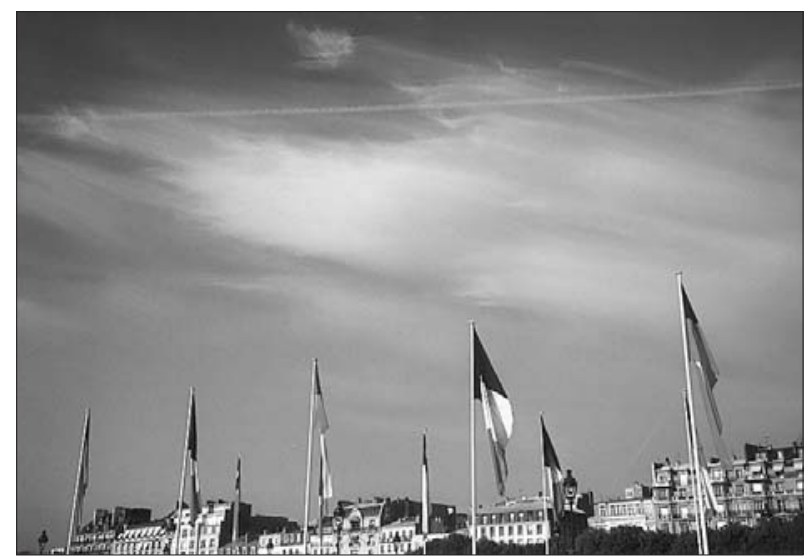

Cirrus et trainée de condensation dans le ciel de Paris. (Photo D. Renaut) cient d'extinction renseigne sur la forme des particules. Par ailleurs, l'hypothèse de sphéricité des particules permet de calculer théoriquement le rapport k en utilisant l'algorithme de Mie. Dans ce calcul, la répartition dimensionnelle des particules est choisie selon les spectres déterminés expérimentalement. Les résultats sont résumés sur la figure 8 , qui représente les valeurs théoriques du rapport $\mathrm{k}$ en fonction du diamètre moyen des particules. Les valeurs expérimentales relatives aux cirrus naturels et aux traînées de condensation sont également reportées sur la figure 8. Les résultats montrent un accord satisfaisant entre les valeurs théoriques et les valeurs déduites expérimentalement et confirment que les traînées de condensation fraîchement formées sont majoritairement composées de particules sphériques.

Ces résultats suggèrent une condensation préalable de gouttelettes d'eau suivie d'une congélation très rapide de celles-ci pour expliquer la formation et l'évolution des trâ̂nées de condensation (Gayet et al., 1996a). Il se produirait ainsi une surproduction de cristaux de glace. Très nombreux comparativement à ceux rencontrés dans les cirrus naturels, ils grossiraient donc plus lentement pour des champs de vapeur d'eau équivalents. Quant aux processus de condensation dans le sillage des avions, ils peuvent s'expliquer par une nucléation hétérogène sur des aérosols produits à l'arrière des moteurs (particules d'acide sulfurique ou de suie).

L'analyse des mesures microphysiques obtenues dans les cirrus et dans les traînées de condensation a également permis de quantifier les limites intrinsèques des appareils de mesure (sondes PMS FSSP et 2D-C, en particulier) pour la caractérisation des cristaux de glace. Des mesures comparatives issues de six sondes $2 \mathrm{D}-\mathrm{C}$ ont clairement mis en évidence des différences importantes sur l'estimation des quantités microphysiques (concentration de particules, contenu en glace, etc.) selon le type d'instrument (Gayet et al., 1993). Ce résultat montre l'impérieuse nécessité de réaliser des vols de comparaison lorsque plusieurs avions sont impliqués dans des expériences coopératives.

Par ailleurs, lorsque les cristaux de glace sont de forme sphérique, la sonde FSSP permet de prendre en compte les particules de diamètre inférieur à $25 \mu \mathrm{m}$, ce qui mène à des rayons effectifs notablement plus petits, avec des concentrations de particules pouvant atteindre 1500 par litre dans les traînées de condensation (Gayet et al., 1996b). En revanche, dans les cirrus naturels où les particules sont majoritairement de forme non sphérique, la sonde FSSP ne permet pas de caractériser les petits cristaux de glace. Cette lacune instrumentale peut entraîner une sous-estimation de plus de $20 \%$ sur les paramètres radiatifs tels que l'albédo et l'émissivité infrarouge (Larsen et al., 1997). Ces résultats soulignent la nécessité de développer un appareil de mesure capable de détecter les cristaux de glace de petite dimension.

Ces dernières années, un effort tout particulier a été porté pour développer les Mesures physico-chimiques mesures de chimie de l'atmosphère. Cette discipline permet d'évaluer la qualité de notre environnement et de nombreux travaux sont engagés dans les domaines du contrôle, de la diffusion et du transport de la pollution. Les échelles à considérer sont très vastes quand il s'agit d'un problème planétaire comme dans le cas du bilan des constituants à effet de serre.

Les mesures physico-chimiques qui sont couramment effectuées à partir des avions concernent les teneurs en oxydes d'azote $\left(\mathrm{NO}, \mathrm{NO}_{2}\right)$, en ozone $\left(\mathrm{O}_{3}\right)$, en dioxyde de soufre $\left(\mathrm{SO}_{2}\right)$, en monoxyde de carbone $(\mathrm{CO})$ et en peroxyde d'hydrogène $\left(\mathrm{H}_{2} \mathrm{O}_{2}\right)$. Il est aussi possible d'effectuer des prélèvements gazeux et des prélèvements de gouttelettes dans les nuages. Les échantillons ainsi prélevés sont ensuite analysés à bord de l'avion ou en laboratoire. Ils permettent en particulier la mesure des concentrations en sulfate de diméthyle (DMS) et en hydrocarbures non méthaniques (NMHC) dans l'atmosphère, après analyse par chromatographie en phase gazeuse, ou la mesure de l'acidité de la pluie après analyse par chromatographie ionique. 


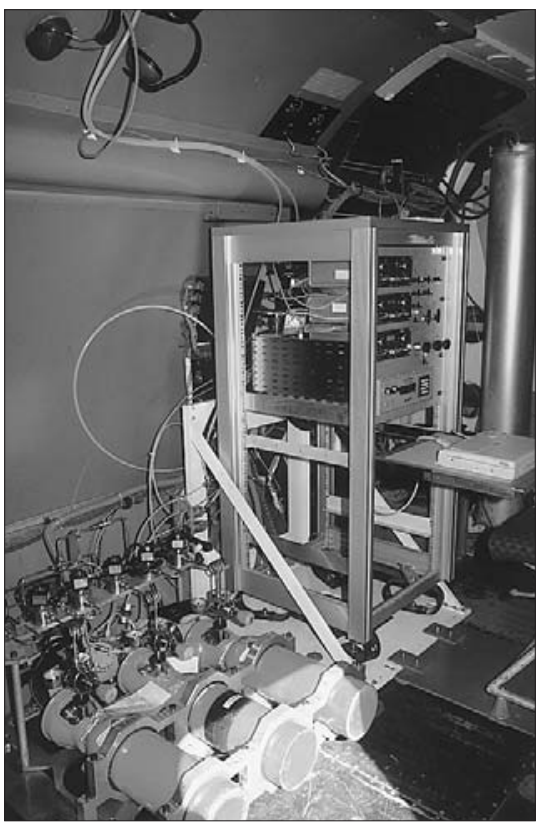

À l'intérieur de l'Arat, l'équipement Licor de I'Onera pour la mesure en continu du CO2 atmosphérique. ( $\bullet$ Insu)
Le cycle planétaire des constituants de l'atmosphère est contrôlé par des sources et des puits locaux qui peuvent être caractérisés en fonction de l'activité des divers écosystèmes rencontrés aux différentes latitudes. L'avion est actuellement le moyen le mieux adapté pour caractériser de telles émissions et de tels puits à une échelle suffisante pour effectuer des bilans. Pour évaluer les flux associés aux divers constituants, il a fallu appliquer à la mesure des composés chimiques les méthodes utilisées jusquelà pour les mesures dynamiques. La méthode de référence pour la mesure des flux repose sur l'analyse de la corrélation entre vitesse verticale de l'air et concentration du constituant chimique transféré. Cette technique nécessite la mesure rapide de concentration des constituants chimiques avec des résolutions temporelles du dixième de seconde. Elle est actuellement réalisable pour des constituants comme l'ozone, le dioxyde de carbone et les aérosols.

La méthode dite «d'échantillonnage conditionnel » (voir encadré ci-dessous) a été développée pour essayer de généraliser les mesures de flux à un grand nombre de constituants en trace de l'atmosphère. Elle ne nécessite pas de mesures rapides de la concentration de l'élément dont on veut connaître le flux. Le principe consiste à prélever, à vitesse ascensionnelle constante, deux échantillons d'air sélectionnés selon le signe de la vitesse verticale de l'air. Le flux vertical d'un composant scalaire s'exprime alors comme une fonction de l'écart de concentration entre les deux échantillons et de l'écart type de la vitesse verticale. Malgré la difficulté rencontrée pour effectuer une mesure précise de la vitesse verticale en temps réel, cette méthode est intéressante car elle évite les difficultés inhérentes aux méthodes classiques de corrélation, à savoir la nécessité d'une mesure à haute résolution temporelle. Son champ d'application le plus évident est la détermination des flux chimiques émis ou fixés par les différents écosystèmes naturels homogènes. Ainsi, Hicks et McMillen (1984) proposent d'utiliser cette méthode pour l'étude du dépôt de polluants. La méthode a été adaptée à la mesure par avion afin de permettre l'évaluation des émissions de constituants en trace de divers écosystèmes homogènes.

\section{Méthode d'accumulation ou d'échantillonnage conditionnel}

C'est une méthode expérimentale simple pour accéder à la mesure directe du flux turbulent. Elle est applicable à la détermination du flux de toute quantité conservative. Elle consiste à réaliser deux fractions d'air échantillonnées suivant la valeur de la vitesse verticale de l'air. La sélection peut être proportionnelle à la vitesse verticale ou simplement dépendante de son signe. Cette dernière méthode, dite "Relaxed eddy accumulation » en anglais, est très simple d'emploi. Le flux vertical est alors déduit de la différence de " concentration » $\Delta \mathrm{X}$ du scalaire étudié entre les deux fractions réalisées et de l'écart type $\sigma_{\mathrm{w}}$ de la vitesse verticale. On montre en effet que le flux peut s'écrire :

$<w X>=\beta \cdot \sigma_{w} \cdot \Delta X$

où $\beta$ est une constante comprise entre 0,58 et 0,62 et qui fait encore, bien sûr, l'objet de nombreuses discussions quant à sa dépendance par rapport aux conditions de turbulence.

Comme le signale cet article, c'est en chimie atmosphérique que les possibilités de cette méthode remarquablement simple s'imposent, en s'appliquant à un très grand nombre de constituants en trace. Elle présente en effet l'avantage de ne nécessiter que la mesure des concentrations moyennes des deux fractions. Le prélèvement étant correctement effectué et les gaz à mesurer éventuellement stabilisés par absorption dans des conteneurs spéciaux, la mesure de l'écart de concentration peut alors être faite tranquillement en laboratoire. Pour calculer le flux, il suffit de connaître en outre l'écart type de la vitesse verticale durant la phase de prélèvement.

Rappelons que cette technique ne peut être appliquée qu'à des systèmes homogènes horizontalement (comme toute mesure de flux vertical turbulent). Elle est particulièrement bien adaptée à la détermination des flux de constituants en traces émis par les écosystèmes homogènes. Quand on l'applique à la mesure aéroportée, cette méthode présente une difficulté puisqu'elle requiert la connaissance, en temps réel et dans l'avion, de la vitesse verticale de l'air. Durant l'expérience Expresso en République centrafricaine, la méthode a été appliquée à la mesure des flux des hydrocarbures non méthaniques émis par la forêt dense humide et par la savane arborée (Greenberg et al., 1997). 


\section{Le lidar Leandre : structure des cellules convectives et vapeur d'eau}

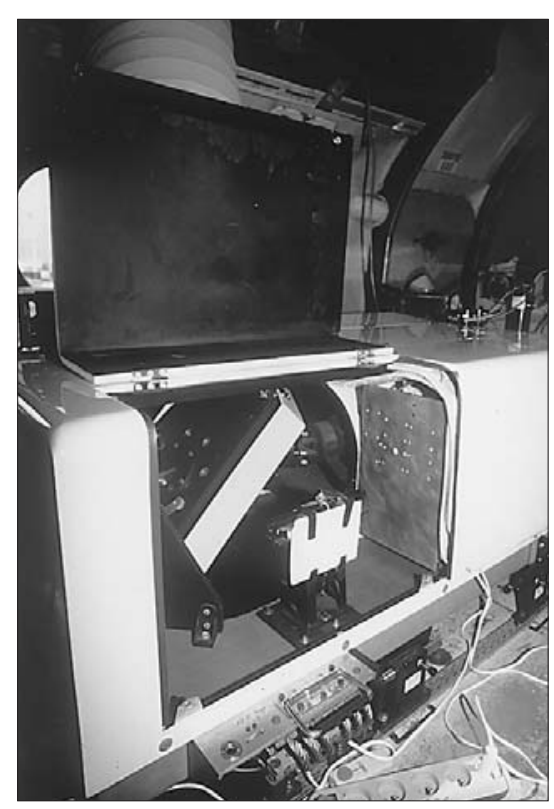

Vue intérieure de l'Arat montrant le lidar aéroporté Leandre. (Photo Météo-France, P. Taburet)

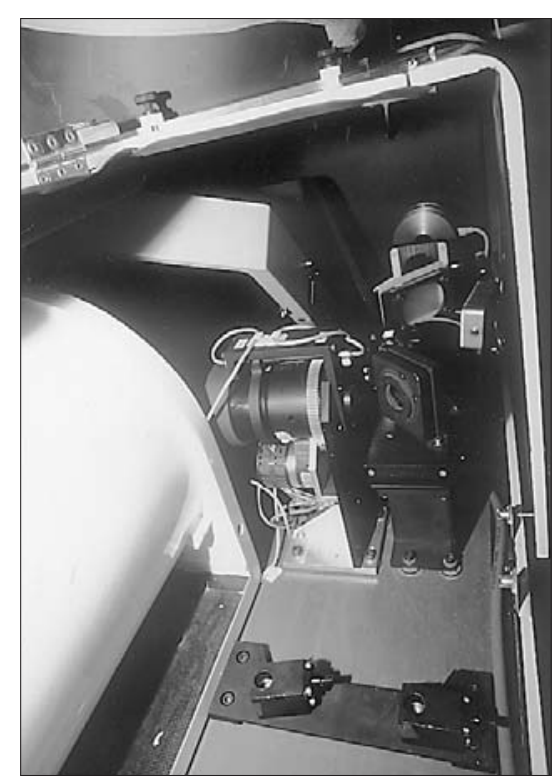

Vue intérieure de l'Arat montrant un détail de I'optique du lidar aéroporté Leandre. (Photo Météo-France, P. Taburet)
Les premiers essais du prototype aéroporté développé à cet effet par le LA et le DT/Insu ont été effectués sur le Fokker 27 au cours de l'expérience Expresso, en 1996 (Druilhet et al., 1996). Deux écosystèmes ont été étudiés: la savane arborée de République centrafricaine et la forêt dense humide du Nord Congo. Ces deux écosystèmes ont un rôle important dans le fonctionnement énergétique de la planète et dans le budget des gaz à effet de serre (cycle de l'ozone). Durant cette expérience, la méthode a été appliquée à la mesure du flux de dioxyde de carbone et des hydrocarbures non méthaniques.

Le programme Leandre (Lidars aéroportés pour l'étude des aérosols, des nuages, de la dynamique, du rayonnement et du cycle de l'eau) est développé conjointement par le CNRS, l'Insu et le Cnes, avec le soutien de Météo-France pour les développements visant la mesure du champ de vent à mésoéchelle (Leandre 3, Wind). Les objectifs concernent la mesure des paramètres dynamiques de la troposphère et l'analyse des propriétés radiatives des nuages et des aérosols. Le programme préfigure également la réalisation de futures expériences spatiales pour des applications à l'échelle du globe et pour l'étude du climat. Les différents développements décrits ici sont l'œuvre d'équipes de projet, regroupant des ingénieurs de plusieurs laboratoires du CNRS dont l'Insu, le LMD et le Service d'aéronomie, et d'équipes scientifiques au sein desquelles un travail important a été effectué par des étudiants en cours de thèse. Un exemple de mesures obtenues par chacun des systèmes actuellement développés (Leandre 1 et 2 ) est présenté ci-après.

Leandre 1 est un lidar à rétrodiffusion utilisant un laser Nd-YAG à impulsion (Quantel) émettant simultanément à $0,532 \mu \mathrm{m}$ et à $1,064 \mu \mathrm{m}$. L'émission laser est polarisée linéairement. Le bloc de détection comporte quatre voies de mesure en détection directe : deux voies à $0,532 \mu \mathrm{m}(0-30 \mathrm{~km})$ pour les lumières polarisées parallèle et perpendiculaire, une voie à $1,064 \mu \mathrm{m}(0-30$ $\mathrm{km})$ et une voie « loupe » $(0,532 \mu \mathrm{m}$ ou $1,064 \mu \mathrm{m})$ à très haute résolution verticale $(1,25 \mathrm{~m})$ pour étudier les interfaces et les zones de transition. Les visées du lidar peuvent s'effectuer, soit au nadir avec une possibilité de balayage transversal sur environ 15 degrés, soit à 20 degrés du zénith en position fixe. Les deux types de configuration peuvent être utilisés au cours d'un même vol sans qu'il soit nécessaire d'intervenir sur l'instrument.

Le principe de la mesure repose sur la diffusion élastique de la lumière par les particules en suspension dans l'atmosphère (diffusion Mie, voir par exemple Van de Hulst, 1957) et les molécules (diffusion Rayleigh). À cela s'ajoute la mesure de dépolarisation de la lumière diffusée, soit par des particules non sphériques (comme les cristaux de glace), soit par diffusion multiple dans les milieux denses comme les nuages. Dans les nuages optiquement minces, le taux de dépolarisation permet de distinguer les phases eau liquide et glace.

La hauteur de la Couche limite atmosphérique (CLA) peut être restituée directement, à partir de l'analyse du signal (Dupont et al., 1994 ; Flamant et Pelon, 1996). Ce point est illustré sur la figure 9 où la valeur du signal rétrodiffusé est représentée en fonction du temps et de l'altitude (120 s correspondant à une distance horizontale de $10 \mathrm{~km}$ environ) en visée au nadir. Ces observations ont été effectuées dans la région des Açores pendant la campagne Semaphore en 1993, grâce aux mesures coordonnées entre l'Arat et le Merlin IV. Le sommet de la CLA est facilement identifiable. Il est marqué par une décroissance rapide du signal résultant du blocage des aérosols qui ne parviennent pas à franchir la région de forte stabilité verticale associée à l'inversion de température. On remarque en fait sur la figure 9 l'existence d'une décroissance rapide du signal à deux niveaux d'altitude. Le premier niveau (1 $000 \mathrm{~m})$ marque le sommet de la couche mélangée en couplage direct avec la surface océanique et correspond à une inversion de température de l'ordre du degré Celsius. Le deuxième (1 800 m) marque la transition vers la troposphère libre et correspond à une inversion plus marquée $\left(6^{\circ} \mathrm{C}\right)$ résultant de la circulation de grande échelle. Ces transitions sont également observées sur le profil d'humidité reporté sur la même figure. En raison de la faible valeur relative de l'inversion, les particules d'air les plus énergétiques peuvent franchir cet obstacle. Lorsqu'une particule atteint le niveau de condensation, marqué par la formation de la base des nuages, l'augmentation de flottabilité associée au dégagement de chaleur latente lui permet d'atteindre 
l'altitude de la deuxième inversion. La figure 9 montre que la hauteur de la couche mélangée est assez élevée, mais également qu'il existe une organisation spatiale de la couche limite instable, à plusieurs échelles d'espace sur l'horizontale. Au sommet de la couche mélangée, le rapport d'aspect apparent des structures nuageuses (rapport de la distance entre nuages à la hauteur de la couche mélangée) est de 2 . Les mesures ont été effectuées dans un axe proche de la direction du vent au-dessus de la couche mélangée. Ce rapport correspondrait alors à une instabilité de type convectif, associée à l'advection, sur cette partie de l'océan, d'air plus froid résultant de la présence d'une dépression centrée sur l'ouest du Portugal.

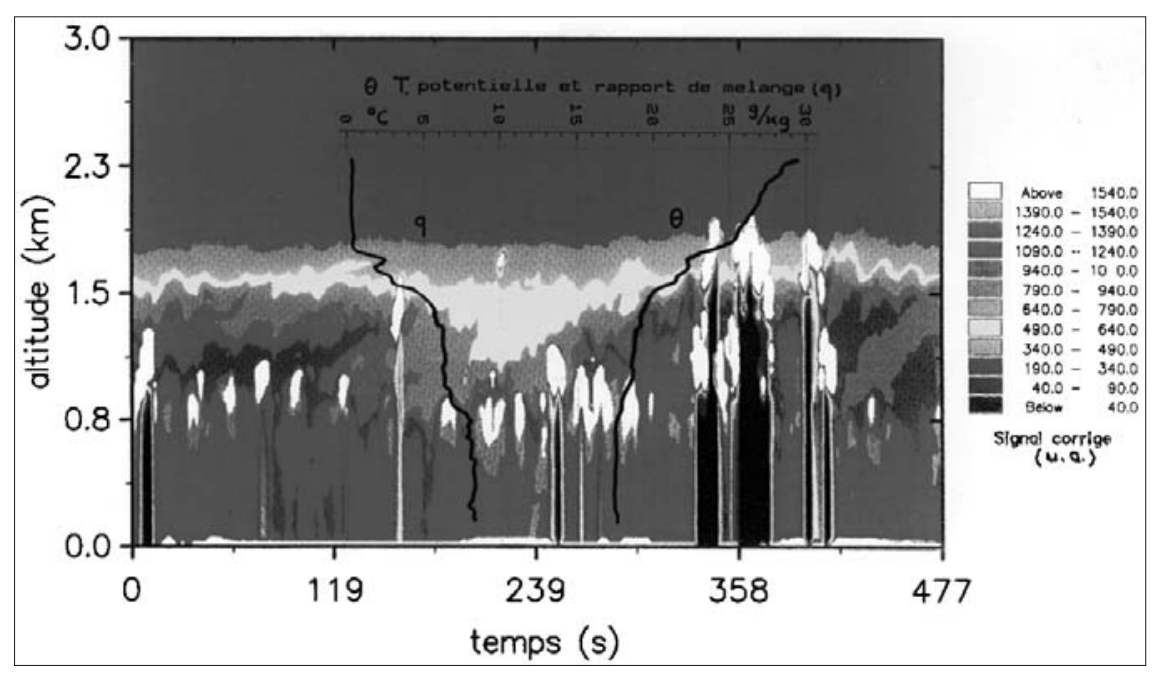

Figure 9 - Exemple de présentation bidimensionnelle du signal lidar rétrodiffusé par les aérosols et les nuages, obtenu par le système Leandre 1 embarqué sur l'avion Arat pendant l'expérience Semaphore. Les profils de température potentielle et de rapport de mélange de la vapeur d'eau mesurés simultanément à partir du Merlin IV sont également représentés (traits pleins).

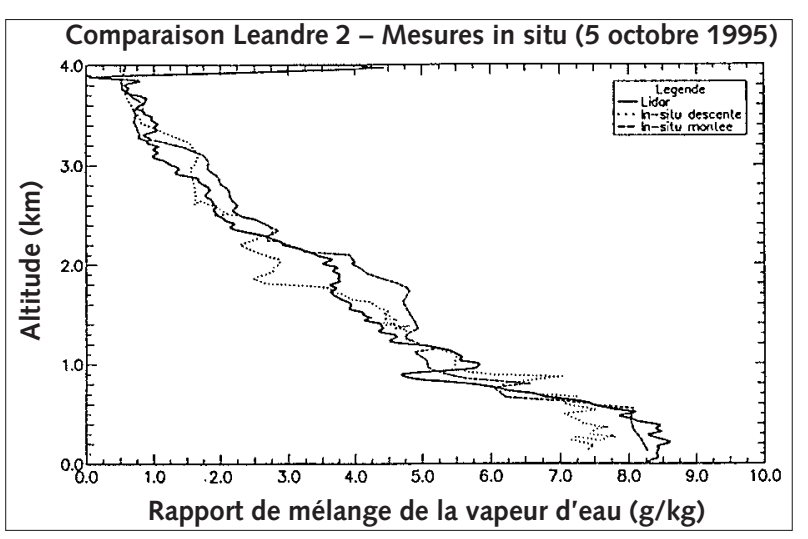

Figure 10 - Comparaison des profils verticaux du rapport de mélange de la vapeur d'eau mesurés par lidar et par capteur in situ à point de rosée. La mesure lidar est effectuée à partir de l'avion Arat stabilisé à $4000 \mathrm{~m}$ au-dessus de la Manche, celle du capteur à point de rosée pendant la montée et la descente de l'avion sur la zone de mesure.
Leandre 2 est un lidar à absorption différentielle qui permet de mesurer les profils verticaux du rapport de mélange de la vapeur d'eau. Les impulsions laser sont émises à deux longueurs d'onde, dont l'une correspond à une raie d'absorption de la vapeur d'eau et l'autre à une raie peu absorbée qui sert de référence atmosphérique. L'étalonnage de l'appareil nécessite l'évaluation de la section efficace de la raie d'absorption par des mesures spectroscopiques indépendantes. La mesure lidar permet alors de déterminer le rapport de mélange de la vapeur d'eau. Les corrections spectrales nécessaires à la détermination précise des profils d'humidité sont effectuées par analyse du signal rétrodiffusé sur la voie de référence et par restitution du profil de diffusion.

La spécificité de ce système réside dans la présence d'une source laser qui utilise un milieu actif solide à alexandrite émettant les deux impulsions requises (séparées de $50 \mu \mathrm{s}$ ) dans un domaine de longueurs d'onde centré autour de $730 \mathrm{~nm}$. Le modèle aéroporté et son contrôle spectral ont été réalisés par l'Insu à partir des prototypes développés au SA dans le cadre du programme « Recherche et technologie » du Cnes (Quaglia et al., 1996).

Les premiers vols de validation de Leandre 2 ont été effectués en septembre 1995 ; un exemple de comparaison avec le profil obtenu par un capteur à point de rosée est présenté sur la figure 10. Les comparaisons montrent que l'écart moyen (ou biais) est inférieur à $0,2 \mathrm{~g} / \mathrm{kg}$ et que, dans la CLA, pour des résolutions verticale de $60 \mathrm{~m}$ et horizontale de $300 \mathrm{~m}$, l'écart type est du même ordre de grandeur. L'utilisation de Leandre 2 est prévue notamment pour l'analyse des échanges de chaleur entre l'océan et l'atmosphère. 


\section{Le radar Ressac: étude des vagues et de la houle}

Les échanges et interactions entre l'océan et l'atmosphère représentent un élément déterminant du climat de la planète et de son évolution. Le radar Ressac a été développé par le Centre d'étude des environnements terrestre et planétaires (CETP) pour la mesure du spectre directionnel des vagues (densité spectrale de la hauteur des vagues en fonction de leur longueur d'onde et de leur direction de propagation). Depuis 1993, il est également utilisé pour la mesure du vent à la surface de l'océan. Il permet de documenter les caractéristiques de la surface océanique (vent et vagues) et de contribuer à la connaissance des échanges océan-atmosphère (Hauser, 1994 ; Eymard et al., 1996), des signatures radar de l'océan (Hauser et al., 1995 ; Caudal et Hauser, 1996 ; Hauser et Caudal, 1996) et de la modélisation et de la prévision des vagues (Lefèvre et al., 1994). C'est un radar fonctionnant à une fréquence de $5,35 \mathrm{GHz}$ avec un système de deux antennes (émission-réception) montées sous le fuselage du Merlin IV et ayant la capacité d'échantillonner sur 360 degrés d'azimut autour de l'axe vertical. Dans le mode utilisé pour la mesure du spectre directionnel des vagues, les vols sont réalisés à $6000 \mathrm{~m}$ d'altitude environ. À partir de la section efficace radar mesurée pour des angles d'incidence compris entre 7 et 21 degrés par rapport à l'axe vertical, le spectre des vagues de grande longueur d'onde (entre 50 et 400 m environ) est déduit dans chaque direction azimutale (Hauser et al., 1992). Le principe de l'analyse consiste à appliquer le modèle linéaire reliant le spectre de modulation du signal reçu dans chaque direction de visée au spectre de pente des vagues. Tous les trente kilomètres environ le long de la trace de l'avion, on obtient un spectre directionnel des vagues. Pour la mesure du vent de surface, des vols en cercle à $2500 \mathrm{~m}$ d'altitude sont réalisés. À partir de la section efficace radar mesurée pour des angles d'incidence compris entre 27 et 41 degrés par rapport à l'axe vertical, on peut obtenir une estimation du module et de la direction du vent de surface en utilisant des modèles empiriques.

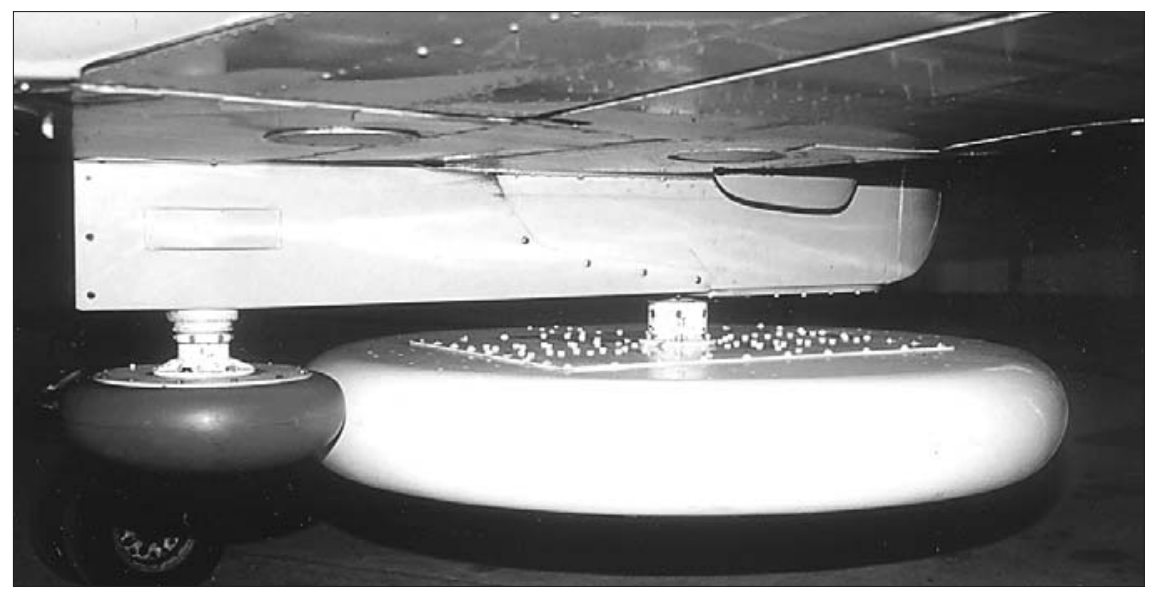

Le radar Ressac a été utilisé dans le cadre de grandes campagnes coopératives internationales (expériences Rene organisées par l'Agence spatiale européenne en 1990 et 1991 ; expérience Swade organisée en 1991 par la Nasa) ou nationales (expérience Semaphore en 1993).

La figure 11 compare un spectre directionnel de vagues obtenu à l'aide du radar Ressac pendant l'expérience Semaphore au spectre correspondant fourni par le modèle VAG de prévision des vagues développé à Météo-France. Pour cette étude, le modèle était initialisé avec les champs de vent issus d'une analyse prenant en compte l'ensemble des données disponibles (bouées, bateaux, satellite ERS 1, etc.). Abstraction faite de l'ambiguïté en direction dans le cas du spectre Ressac, on constate un bon accord entre les deux spectres a et b, avec une composante de houle (fréquence dominante $0,08 \mathrm{~Hz}$ ) se propageant vers le sud-est et une composante de « mer du vent » de fréquence plus élevée se propageant vers le nord. On a pu améliorer notablement le modèle VAG grâce aux comparaisons systématiques de ses résultats avec les observations du radar Ressac. 
Figure 11 - Spectres directionnels des vagues obtenus pendant la campagne Semaphore, le 20 octobre 1993.

a, spectre mesuré à l'aide du radar aéroporté Ressac. Les contours donnent la densité spectrale de hauteur de vagues, normalisée par rapport au pic du spectre, en fonction de la fréquence des vagues (distance par rapport au centre de la figure entre 0 et $0,25 \mathrm{~Hz}$ )

et de la direction de propagation (le nord étant représenté vers le haut de la figure). Le spectre obtenu présente une ambiguïté de 180 degrés sur la direction de propagation, ambiguité inhérente au principe de la mesure.

$b$, même représentation que $a$, mais pour le spectre déduit du modèle VAG de prévision des vagues.
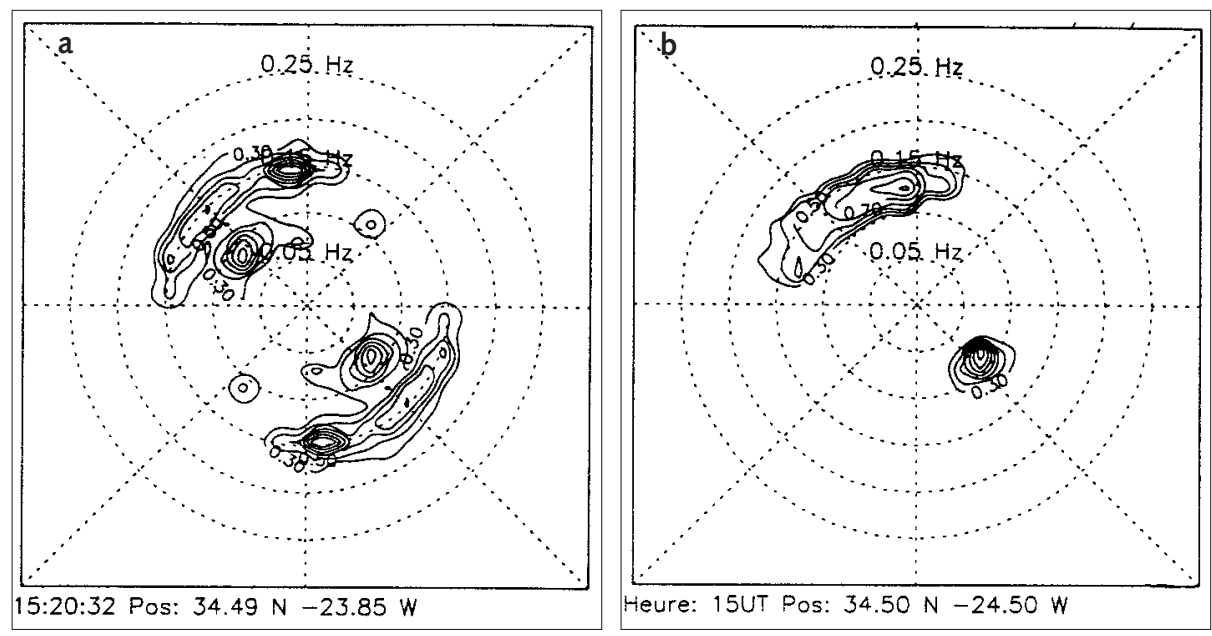

L'instrument Polder

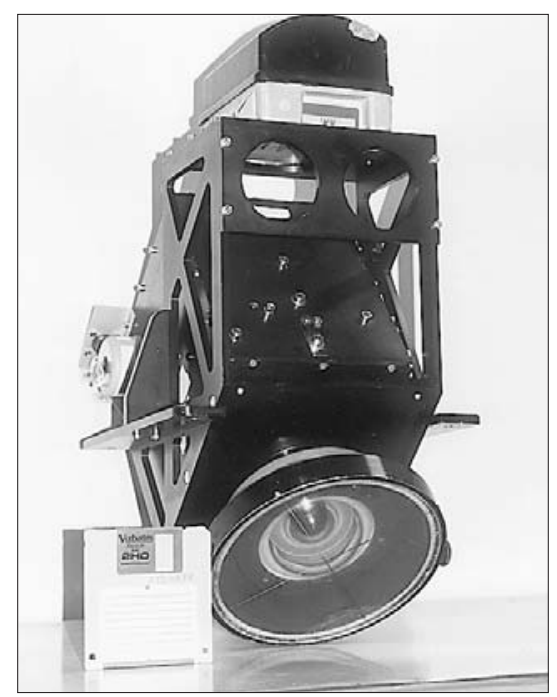

Tête optique de l'instrument Polder en version aéroportée " inclinée ». ( $\bullet$ J.-Y. Balois et O. Boucher, LOA/CNRS/USTL)

Dans les nuages

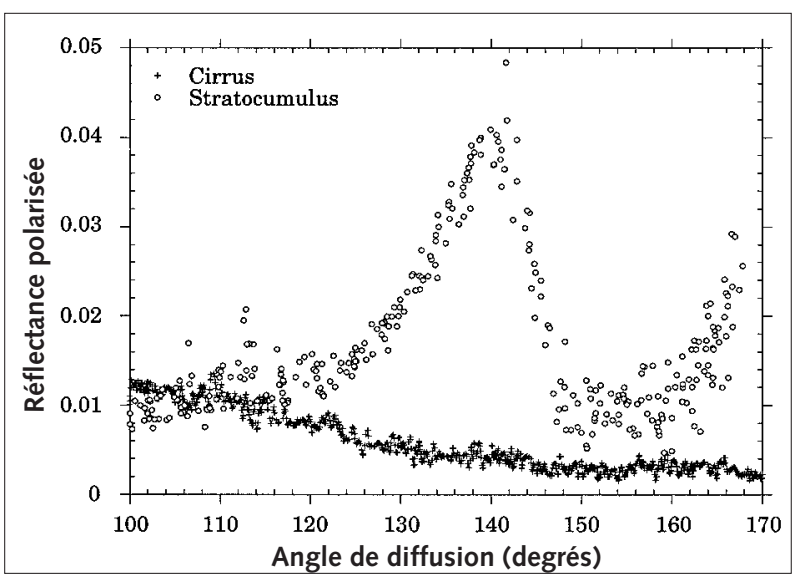

Figure 12 - Réflectance polarisée mesurée par Polder à 865 nm en fonction de l'angle de diffusion pour les nuages constitués d'eau liquide (stratocumulus) et ceux constitués de cristaux de glace (cirrus).
Polder (Polarization and Directionality of Earth Reflectances) est un instrument qui mesure la directionnalité et la polarisation du rayonnement solaire réfléchi par le système Terre-atmosphère (Deschamps et al., 1994). Ces paramètres conduisent à l'évaluation de nombreuses variables décrivant l'état de l'océan, des terres émergées, des aérosols et des nuages. Polder a été embarqué en août 1996 sur le satellite japonais à orbite polaire Adeos et a fourni huit mois de mesures avant la perte de la plate-forme en juillet 1997. Au vu des premières analyses, les données obtenues permettront de démontrer le caractère innovant et l'intérêt scientifique et technique de la mission, même si certains des objectifs nécessitant de disposer de séries pluriannuelles seront de toute évidence pénalisés. Afin de préparer cette mission, un simulateur aéroporté a d'abord été développé au Laboratoire d'optique atmosphérique (LOA) de Lille. L'appareil est constitué d'un objectif télécentrique à large champ ( \pm 57 degrés) associé à une matrice CCD de 288 x 384 pixels. Neuf canaux sont disponibles dans le domaine spectral 400-950 nm. Il existe des roues porte-filtres adaptées aux différents objectifs de la mission, à savoir l'étude des aérosols et des surfaces terrestres (campagnes de La Crau en 1990 et 1991, des Landes en 1990, d'Orgeval en 1991, Hapex-Sahel au Niger en 1992 et Boreas au Canada en 1993), l'étude des surfaces océaniques et des aérosols marins sur la Méditerranée (campagne Medimar en 1991) et dans l'Antarctique (campagne Racer en 1991), et enfin l'étude des nuages (les nuages bas lors de Sofia-Astex aux Açores en 1991, les nuages moyens lors de Cleopatra en 1991 et les nuages hauts lors d'Eucrex en 1994). Quelques exemples des paramètres auxquels Polder permet d'accéder sont fournis ci-après.

L'épaisseur optique des nuages peut être déduite des mesures de Polder en employant l'approximation dite « plan-parallèle ». Cette hypothèse a pu être validée pour les nuages bas grâce aux données acquises pendant Sofia-Astex (Weill et al., 1995). En effet, les données de Polder ont montré que les réflectances bidirectionnelles des scènes totalement nuageuses se comparent assez précisément (2 à $3 \%$ d'écart) à celles de couches « plan-parallèle » hétérogènes (Descloitres et al., 1994).

En ce qui concerne les nuages hauts de type cirrus, on a montré, durant Eucrex 94, qu'un modèle de cristaux de glace hexagonaux rend bien compte de la fonction de distribution des réflectances bidirectionnelles, ce qui permet de définir un modèle radiativement équivalent pour les cirrus (Chepfer et al., 1996).

Grâce aux campagnes réalisées sur les différents types de nuages, on a pu montrer (fig. 12) que la polarisation de la lumière réfléchie par les nuages permettait de déterminer la phase de l'eau condensée : glace ou eau liquide (Goloub et al., 1994). On a aussi montré que Polder pouvait déterminer l'altitude des nuages en utilisant, soit l'absorption par l'oxygène (Bréon et Bouffiès, 1996), soit la polarisation du signal moléculaire (Parol et al., 


\section{Au-dessus de l'océan}

\section{Au-dessus des terres émergées}

1996). La synthèse de ces campagnes aéroportées a également permis de mettre au point les algorithmes qui seront employés pour obtenir le bilan radiatif terrestre à partir des données Polder dans sa version embarquée sur Adeos (Buriez et al., 1997).

La mesure de la polarisation permet une meilleure caractérisation des aérosols et, par voie de conséquence, une meilleure correction des effets atmosphériques pour les mesures de couleur de l'océan. En effet, la contribution de l'océan ne représente que quelques pour cent (moins de $10 \%$ en moyenne) du signal mesuré par le capteur ; la précision avec laquelle sont restituées les réflectances marines, interprétées en termes de concentration en phytoplancton, en matière organique dissoute ou en sédiments, dépend largement de ces corrections. Les premières mesures aéroportées Polder acquises au-dessus de l'océan (campagne Medimar 1991) ont pu être interprétées à l'aide d'un modèle analytique très simple de réflectance bidirectionnelle (Bréon et Deschamps, 1993). Ce modèle, bien que peu précis pour des mesures de couleur de l'océan, permet de bien reproduire les signatures directionnelles. En particulier, la distribution angulaire de la réflexion du rayonnement solaire par la surface des vagues, distribution liée à la direction et à la vitesse du vent, est parfaitement reproduite. L'expérience Racer 1991 a permis d'appliquer les algorithmes de détermination des aérosols (Deuzé et al., 1992) et de couleur de l'océan (Frouin, 1992) à des mesures réelles, mais n'a pu être pleinement exploitée à cause de problèmes de lumière parasite apparus au cours de la campagne.

Les réflectances directionnelles déduites des mesures Polder permettent une meilleure caractérisation des propriétés de surface. La polarisation permet de séparer le signal atmosphérique du signal provenant de la surface, et donc d'observer les aérosols au-dessus des continents. Ces capacités de l'instrument ont été illustrées à l'aide des mesures acquises pendant les expériences Crau, Hapex et Boreas. La réflectance dans le canal à $650 \mathrm{~nm}$ (fig. 13) montre en effet la variabilité spatiale des réflectances de surface contaminées par la présence de l'atmosphère, alors que la même scène observée en lumière polarisée montre un signal indépendant des propriétés de surface, ce qui permet de remonter aux aérosols atmosphériques (Deuzé et al., 1993 ; Herman et al., 1997). Les signatures directionnelles des surfaces ont également été utilisées pour l'inversion d'un modèle de réflectance de surface. On a pu ainsi quantifier, cartographier et modéliser l'amplitude des signatures directionnelles des réflectances des surfaces naturelles à l'aide d'un modèle simple (Leroy et Bréon, 1996). Les mesures Polder permettent par ailleurs d'estimer la vapeur d'eau atmosphérique à l'aide d'une technique d'absorption différentielle : les études théoriques montrent une précision accessible de l'ordre de $10 \%$; les données aéroportées obtenues pendant les campagnes Hapex, Boreas et Eucrex 94 ont permis de valider la méthode et de confirmer ces résultats (Bouffiès et al., 1997).
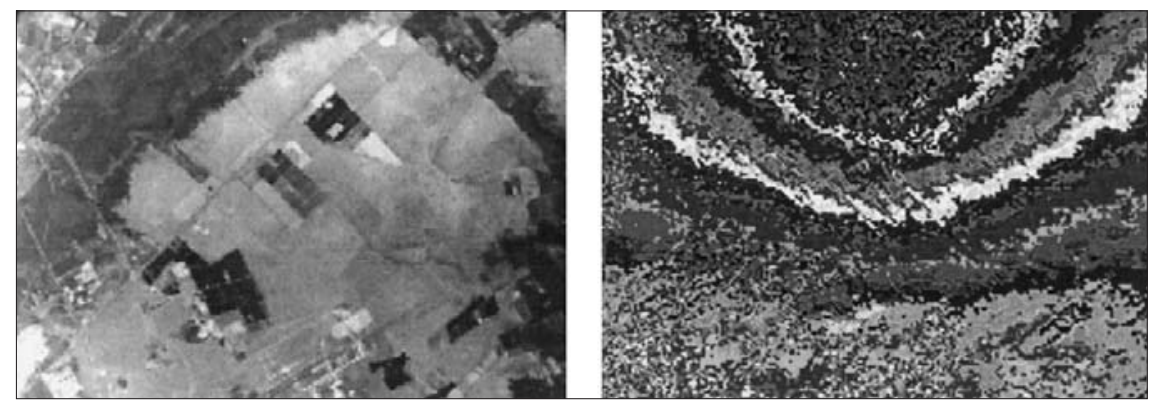

Outre sa capacité à fournir des mesures par tous temps (transparence des nuages), le rayonnement micro-ondes émis par la surface terrestre est sensible à la quantité d'eau contenue dans la végétation ou à la surface du sol, par le biais des variations de la constante diélectrique (Kerr et Njoku, 1990 ; Kerr et Wigneron, 1995 ; Raju et al., 1995). À certaines fréquences situées autour du pic d'absorption de la vapeur d'eau $(22,235 \mathrm{GHz})$, le rayonnement reçu au-dessus d'une partie de l'atmosphère peut être utilisé pour déterminer le contenu en eau atmosphérique intégré. De même, le canal $90 \mathrm{GHz}$ peut servir pour déterminer les précipitations ou pour des études sur la neige et la glace. 
Portos est un radiomètre profileur micro-ondes réalisé en 1991 par Matra Marconi System, sous maîtrise d'œuvre du Centre national d'études spatiales. Il peut fonctionner en version sol sur une grue (Wigneron et al., 1995 ; Wigneron et al., 1996), un portique ou un téléphérique (Sherjal et Fily, 1994), ainsi qu'en version aéroportée (Grosjean et Sand, 1994). Nous nous intéresserons ici à la version aéroportée dont la principale différence avec la version sol est l'absence du canal 1,4 GHz (21 cm, bande L), pour des raisons d'encombrement de l'antenne. La version aéroportée comporte cinq fréquences $(5 ; 10,7 ; 23,8$; 36,5 et $90 \mathrm{GHz}$ ), toutes polarisées. Le faisceau peut être orienté dans le plan de l'axe de vol afin de donner un angle de visée entre 0 et 50 degrés vers l'arrière de l'appareil. La résolution au sol de l'instrument est liée à la largeur du faisceau à demi-puissance, qui est en moyenne de 11 degrés. L'instrument est un profileur, c'est-à-dire qu'il n'acquiert qu'un seul point perpendiculairement à l'axe de vol. En général, Portos est utilisé dans des configurations de vol à basse altitude (900 m) avec un angle de visée de 45 degrés, ce qui donne une résolution de l'ordre de $300 \mathrm{~m}$, ou bien à altitude moyenne $(5000 \mathrm{~m})$ avec un angle de visée proche de 0 degré, ce qui donne une résolution au sol de l'ordre de $2 \mathrm{~km}$. Pour réaliser des cartographies, les plans de vol sont constitués d'axes parallèles (une dizaine), espacés d'une distance identique à la résolution au sol.

Le radiomètre Portos a été conçu pour fournir des jeux de mesures passives en micro-ondes permettant la mise au point de techniques et d'algorithmes d'inversion de paramètres de la surface. C'est un instrument possédant à peu de choses près les caractéristiques du radiomètre ATSR/M (Along-Track

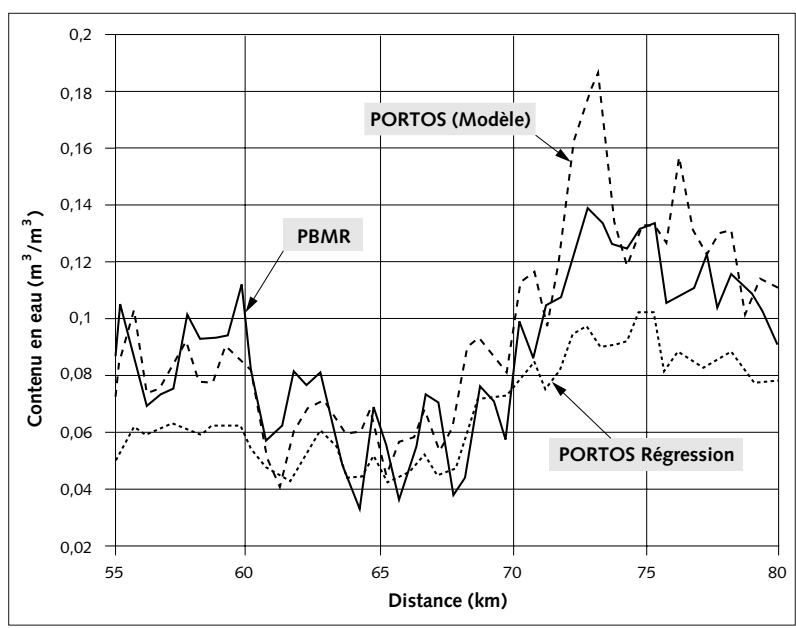

Figure 14 - Estimation, lors de la campagne Hapex-Sahel, de I'humidité superficielle au moyen de mesures micro-ondes le long d'un transect est-ouest couvrant les sites centraux est et ouest (jour $256,1992)$. La courbe en trait plein correspond au radiomètre PBMR $(1,4 \mathrm{GHz})$ donnant la « vérité » terrain. En tiretés, l'estimation obtenue avec le radiomètre Portos $(5 \mathrm{GHz})$ en applicant un modèle inverse prenant en compte la végétation. En pointillés, l'estimation obtenue à partir d'une régression linéaire entre les données Portos et les mesures au sol. (D'après Chanzy et al., 1997) Scanning Radiometer/Microwave) et du futur radiomètre MIMR (Multispectral Imaging Microwave Radiometer) qui doivent être embarqués sur les satellites défilants Metop, EOS et Adeos. Un tel radiomètre n'existait pas avant Portos.

Portos a été utilisé pour la première fois dans sa version aéroportée dans le cadre de l'expérience Hapex-Sahel (Goutorbe et al., 1994 ; Prince et al., 1995). Les principaux axes de recherche concernaient la détermination de l'humidité de surface, du contenu en eau de la végétation et de la température de surface. Il était également prévu d'étudier les relations d'échelle entre les acquisitions ponctuelles au sol, les acquisitions Portos à deux résolutions $(300 \mathrm{~m}$ et $2 \mathrm{~km})$ et les acquisitions satellitaires $(50 \mathrm{~km})$. Portos a aussi été utilisé (campagnes Crau, Murex et Forêt des Landes) pour le suivi de l'eau superficielle et pour déterminer le réservoir profond.

La figure 14 montre un exemple de résultat obtenu au moyen de Portos lors de la campagne Hapex-Sahel (Chanzy et al., 1997). Bien que le canal à $5 \mathrm{GHz}$ ne soit pas le plus approprié pour la détermination de l'humidité de surface, nous pouvons constater une très bonne estimation lorsque cette dernière est comparée aux données du radiomètre micro-ondes PBMR $(1,4 \mathrm{GHz})$, surtout lorsque la végétation est prise en compte. Étant calée sur des mesures au sol et un modèle simple d'émission, la mesure PBMR est ici considérée comme faisant référence. Lors de cette campagne, des travaux ont également montré qu'il était possible de quantifier la biomasse et la température de surface au moyen des données Portos en faisant usage des différentes fréquences (Calvet et al., 1996 ; Wigneron et al., 1997).

\section{Le radiomètre} Push-Broom et l'étude des peuplements de pins
L'instrument Push-Broom est un radiomètre optique à balayage électronique disposant des quatre bandes spectrales du satellite Spot. Il est notamment utilisé pour définir les futures missions spatiales avec des capteurs optiques à très haute résolution. L'étude présentée ici, réalisée dans le cadre de travaux préparatoires du Cnes relatifs à l'instrument HRG (Haute résolution géométrique) qui devrait équiper les futures générations de Spot, concerne l'application au domaine forestier (Riom et Guyon, 1992). Un des objectifs est de rechercher la précision géométrique optimale, en vue d'utiliser de l'information provenant de la texture pour mieux différencier les types de peuplement (classes d'âge, de densité, etc.) et bien détecter les coupes d'éclaircies. Plusieurs résolutions spatiales, de quelques 


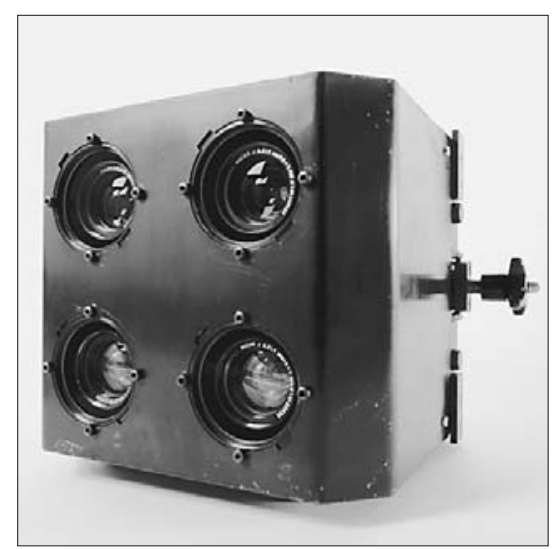

Tête optique du radiomètre Push-Broom avec ses quatre canaux spectraux. ( $\bullet$ Cnes) mètres à quelques dizaines de mètres, sont comparées, l'évaluation, fondée sur l'analyse de la texture des images, incluant la stéréoradiométrie.

Les peuplements de pins présentent des propriétés spectrales voisines quelle que soit la résolution spatiale, mais une très forte dynamique de radiométrie. La texture des images est liée à la structure forestière (figure 15) ; ainsi, les écarts d'un pixel à l'autre sont beaucoup plus élevés sur le peuplement B, de structure très hétérogène, que sur le peuplement $\mathrm{E}$, plus homogène. L'étude montre qu'après dégradation de la résolution, la structuration spatiale de la radiométrie observée à 1,67 m est assez bien conservée jusqu'à $5 \mathrm{~m}$. Et même jusqu'à $10 \mathrm{~m}$ dans le proche infrarouge, la texture des peuplements reste porteuse d'une information fine sur leur structure.

Pour les peuplements de pins étudiés, si la limite de résolution géométrique est inférieure à $10 \mathrm{~m}$, la texture d'image dans le visible est révélatrice de la répartition spatiale des pins, à condition que la dynamique des niveaux radiométriques ne soit pas trop faible. Cette résolution optimale est vraisemblablement plus proche de $5 \mathrm{~m}$ que de $10 \mathrm{~m}$.

Les trois avions arrivent en fin de potentiel et devront être remplacés d'ici trois ou quatre ans.

La communauté scientifique impliquée par l'utilisation et l'exploitation des mesures aéroportées est estimée à plus de 200 chercheurs et ingénieurs appartenant au CNRM, au CNRS, à l'Enseignement supérieur, à l'Inra, à l'Ifremer, au Cnes, au Shom... La plupart des grands programmes nationaux interorganismes (Programme national atmosphère et océan à moyenne échelle [Patom], Programme national de chimie atmosphérique [PNCA], Programme national d'étude de la dynamique du climat [PNEDC], Programme national de recherche en hydrologie [PNRH], Programme national de télédétection spatiale [PNTS], etc.) sont directement concernés par l'utilisation des moyens aéroportés. Les besoins de cette communauté ont été clairement exprimés à travers une enquête organisée par le Comité scientifique des avions de recherche atmosphérique et de télédétection et à l'occasion du colloque de prospective organisé en octobre 1993, à Hourtin, par la Commission scientifique océan-atmosphère de l'Insu. Les opérations de recherche stratosphérique nécessitant le recours à des avions capables de voler très haut, notre prospective a été limitée aux besoins associés à l'étude de la troposphère et de ses échanges avec la stratosphère ou avec la surface terrestre. Les travaux des groupes d'experts mis en place par le Comité
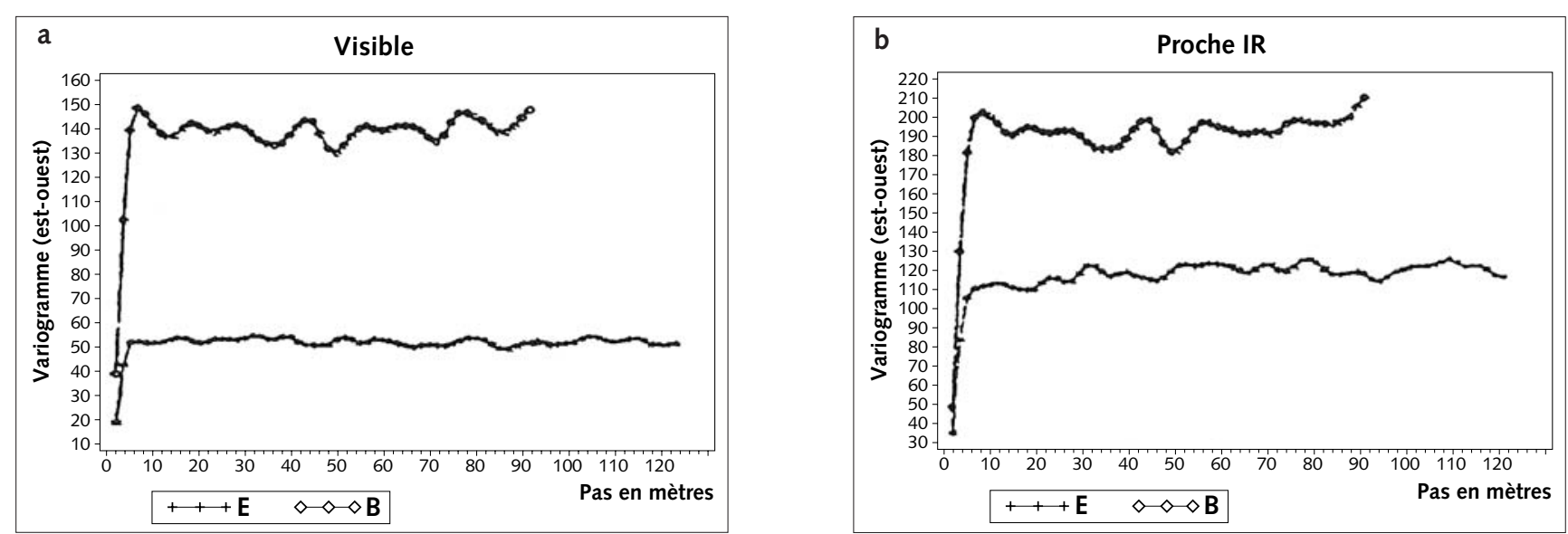

Figure 15 - Variogrammes de la radiométrie suivant une direction de visée est-ouest pour une résolution spatiale de 1,67 m (données rectifiées), en fonction de la texture d'image de deux peuplements adultes de pins:

- peuplement B : 47 ans, structure hétérogène, issu d'un semis naturel ;

- peuplement $E$ : 35 ans, structure homogène, issu d'un semis artificiel en rangs est-ouest.

(D'après Riom et Guyon, 1992) 
L'instrumentation

\section{Les missions et les contraintes}

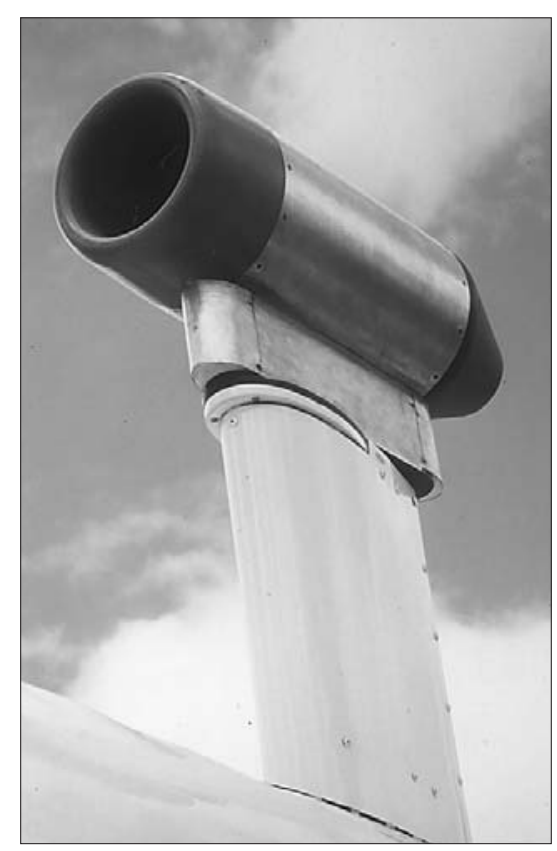

scientifique ont abouti à la rédaction d'un document intitulé « Rapport de prospective sur les futurs avions de recherche troposphérique », qui propose une analyse des besoins futurs et des choix possibles pour répondre à ces besoins.

Les thèmes qui devraient bénéficier en premier lieu de l'utilisation des futurs avions instrumentés sont l'étude de l'évolution climatique, la dynamique atmosphérique et météorologique, l'océanographie et le couplage océan-atmosphère, les surfaces continentales et les échanges sol-atmosphère. À côté des domaines traditionnels (dynamique atmosphérique, physique des nuages, échanges entre la surface terrestre et l'atmosphère), on peut s'attendre à une très forte augmentation des demandes venant de la physico-chimie atmosphérique, de l'océanographie, de la validation des mesures satellitaires et de l'emploi des avions comme plates-formes d'essai des instruments destinés à être embarqués sur satellite.

La réalisation des programmes futurs nécessite l'embarquement de toute une panoplie de capteurs pour effectuer les mesures classiques des paramètres dynamiques et thermodynamiques, des caractéristiques des nuages, mais aussi pour renforcer les mesures de chimie atmosphérique (mesure in situ des gaz traces par chimiluminescence, par chromatographie, par fluorescence ou par diodes laser, mesure de profils d'aérosols ou d'ozone à l'aide des lidars Leandre 1 et Alto, prélèvements isocinétiques pour des analyses en temps différé...), d'électricité atmosphérique (moulins à champs, anneaux d'induction, capteurs de charge d'espace...), de profils atmosphériques (radiomètres Portos, Iris, Marss...) et d'état de surface (radiomètres Push Broom, Casi, Polder, Barnes, radar Ressac...). Dans les années qui viennent, de nombreux capteurs en cours de développement vont devenir opérationnels et offrir de nouvelles possibilités. On peut citer en particulier :

- le système Rali (Radar et lidar) qui associe un radar millimétrique et un lidar bifréquence pour l'étude microphysique et radiative des structures nuageuses ;

- le lidar Doppler Wind pour la restitution des champs tridimensionnels de vent ;

- le démonstrateur Iasi (Interféromètre atmosphérique pour le sondage infrarouge) pour le sondage à haute résolution verticale de la structure thermodynamique de l'atmosphère et la mesure des constituants minoritaires dans la troposphère ;

- les sondes largables (encore appelées « dropsondes »).

À l'exemple du partage des tâches entre le Fokker 27 et le Merlin IV qui s'est produit dans un grand nombre d'expériences (Sofia-Astex, Hapex-Sahel, Eucrex, Semaphore, Pyrex...), les missions et contraintes visant à répondre aux besoins futurs conduisent à une stratégie de mesure coordonnée à deux avions.

L'utilisation des avions actuels a été fortement limitée par deux contraintes, à savoir leur faible autonomie et leur plafond peu élevé. Les performances minimales nécessaires pour répondre de façon satisfaisante aux besoins sont :

- une autonomie pleine charge d'au moins 8 heures, permettant de réaliser des missions au-dessus de l'océan et d'échantillonner des systèmes de grande dimension, tels que les systèmes frontaux ;

- une aptitude à voler près de la surface (à une altitude d'environ 50 m), afin d'accéder aux échanges sol-atmosphère et océan-atmosphère ;

- un plafond de vol proche de la tropopause, soit 9 à $10 \mathrm{~km}$ aux latitudes moyennes, pour l'étude des cirrus et des échanges troposphère-stratosphère ;

- une vitesse de vol pouvant être réduite à moins de $350 \mathrm{~km} / \mathrm{h}$ pendant les opérations mettant en œuvre certains capteurs comme les spectromètres de gouttes de nuage ;

- une charge utile ( 2 tonnes pour le plus petit et 4 tonnes pour le plus gros) permettant en particulier d'embarquer des instruments de télédétection et de physico-chimie, lourds et encombrants ;

- une structure permettant l'implantation d'antennes pour les radars et de fenêtres de visée pour les radiomètres, les spectromètres et les lidars ;

- un maximum de souplesse d'utilisation et un faible coût d'immobilisation.

Vue extérieure montrant, fixé sur le dessus du fuselage du Merlin IV, le thermo-anémomètre sonique développé par le LMD. (Photo Météo-France, CNRM/CAM) 


\section{Le choix des futurs avions}

\section{Remerciements}

\section{BIBLIOGRAPHIE}

Au moment d'effectuer un choix pour le remplacement de nos avions, il est intéressant de regarder les solutions adoptées par nos partenaires scientifiques. Pour répondre aux besoins de la recherche atmosphérique, les Américains ont choisi d'équiper une flotte de plus de trente avions dont plusieurs admettent une charge utile supérieure à 5 tonnes (10 vecteurs), une altitude maximale supérieure à $10 \mathrm{~km}$ (14 vecteurs), une autonomie supérieure à 9 heures (11 vecteurs) et une distance de vol supérieure à $5600 \mathrm{~km}$ (12 vecteurs). Du côté européen, les Britanniques ont un porteur (le C130) répondant à l'ensemble des performances présentées ci-dessus et deux avions de plus petite taille, alors que les Allemands ont choisi de s'appuyer sur toute une flotte d'avions instrumentés (actuellement neuf avions) et sur le développement (encore hypothétique) d'un avion spécialement adapté aux altitudes élevées : le Strato-2C (aujourd'hui, ce programme est apparemment abandonné).

Pour répondre aux besoins de la communauté scientifique française, on suggère de remplacer les trois avions français actuels par deux avions de caractéristiques complémentaires (mesures in situ et télédétection) correspondant à des avions du type ATR 42 ou Dornier 328 pour l'avion 2 tonnes et du type ATR 72 ou Saab 2000 pour l'avion 4 tonnes.

Des informations supplémentaires ont été obtenues des constructeurs de ces avions sur la faisabilité et le coût des transformations nécessaires pour installer l'instrumentation de recherche atmosphérique et de télédétection. Le dossier est maintenant en cours d'instruction auprès des ministères de tutelle.

Nous souhaitons ici remercier P. Bessemoulin et G. Duverneuil (MétéoFrance/CNRM), ainsi que T. Phulpin (Centre national d'études spatiales) pour leurs remarques et contributions.

Une liste complète des documents relatifs à l'instrumentation et aux modes d'utilisation des avions, ainsi que des articles scientifiques mettant en valeur l'analyse des données recueillies pendant des campagnes de mesure, est disponible auprès de :

Insu/DT

77, avenue Denfert-Rochereau

75014 Paris

Attié J.-L., 1994 : Étude d'un écoulement près d'un relief de moyens aéroportés (Expérience PYREX). Thèse de l'université Paul Sabatier nº 1694.

Attié J.-L., A. Druilhet, P. Durand et B. Benech, 1997 : Two-dimensional structure of mountain waves observed by aircraft during PYREX experiment. Ann. Geophysicae, sous presse.

Baker B., J.-L. Brenguier et W. Cooper, 1998 : Sources of radar reflectivity in small cumulus clouds: Theory and measurements. Soumis à J. Appl. Meteor.

Bouffiès S., F.-M. Bréon, D. Tanré et P. Dubuisson, 1997 : Atmospheric water vapor estimate by a differential absorption technique with the POLDER instrument. J. Geophys. Res., 102, 3831-3841.

Bougeault P., B. Benech, P. Bessemoulin, B. Carissimo, A. Jansa, J. Pelon, M. Petitdidier et E. Richard, 1996 : Les résultats de Pyrex. La Météorologie 8 série, 16, 11-30.

Brenguier J.- L. et L. Amodei, 1989 : Coincidence and dead-time corrections for the particle counters. Part I: A general mathematical formalism. J. Atmos. Ocean. Technol., $6,575-584$.

Brenguier J.-L., 1989 : Coincidence and dead-time corrections for the particle counters. Part II: High concentration measurements with an FSSP. J. Atmos. Ocean. Technol., 6, 585-598.

Brenguier J.-L., 1990 : Parameterization of the condensation process in small non-precipitating cumuli. J. Atmos. Sci., 47, 1128-1148.

Brenguier J.-L., 1991 : Parameterization of the condensation process: A theoretical approach. J. Atmos. Sci., 48, 264-282.

Brenguier J.-L., A. R. Rodi, G. Gordon et P. Wechsler, 1993 : Real time detection of performance degradation of the Forward Scattering Spectrometer Probe. J. Atmos. Ocean. Technol., 10, 27-33. 
Brenguier J.-L., 1993 : Observations of cloud microstructure at the centimeter scale. J. Appl. Meteor., 32, 783-793.

Brenguier J.-L., D. Baumgardner et B. Baker, 1994 : A review and discussion of processing algorithms for FSSP concentration measurements. J. Atmos. Ocean. Technol., 11, 1409-1414.

Brenguier J.-L. et F. Burnet, 1996 : Experimental study of the effect of mixing on droplet spectra. 12th Int. Conf. on Clouds and Precip. Zurich, août 1996, 67-70.

Brenguier J.-L. et L. Chaumat, 1996 : Condensational droplet growth in cumulus clouds. 12th Int. Conf. on Clouds and Precip. Zurich, août 1996, 57-60.

Brenguier J.-L. et L. Chaumat, 1998 : Droplet spectra broadening in convective clouds. Soumis à J. Atmos. Sci.

Brenguier J.-L., T. Bourrianne, A. Coelho, J. Isbert, R. Peytavi, D. Trevarin et P. Wechsler, 1998a : Improvements of droplet size distribution measurements with the Fast-FSSP. J. Atmos. Ocean. Technol., sous presse.

Brenguier J.-L., H. Pawlowska, L. Schueller et Y. Fouquart, 1998b : Radiative properties of boundary layer clouds: Optical thickness and effective radius versus geometrical thickness and droplet concentration. Soumis à J. Atmos. Sci.

Bréon F.-M. et P.-Y. Deschamps, 1993 : Optical and physical parameter retrieval from POLDER measurements over the ocean using an analytical model. Remote Sens. Environ., 43, 193-207.

Bréon F.-M. et S. Bouffiès, 1996 : Land surface pressure estimate from measurements in the oxygen A absorption band. J. Appl. Meteor., 35, 69-77.

Buriez J.-C., C. Vanbauce, F. Parol, P. Goloub, M. Herman, B. Bonnel, Y. Fouquart, P. Couvert et G. Seze, 1997: Cloud detection and derivation of cloud properties from POLDER. Int. J. Remote Sensi., 18, 1, 2785-2813.

Calvet J.-C., A. Chanzy et J.-P. Wigneron, 1996 : Surface temperature and soil moisture retrieval in the Sahel from airborne multifrequency microwave radiometry. IEEE Trans. Geosci. Remote Sens., 34, 588-600.

Campistron B., F. Lohou, A. Druilhet et P. Foster, 1995 : Investigation on the dynamics of organized clear air planetary boundary layer with Doppler radar and aircraft measurements. 27th Conference on Radar Meteorology. Vail, Colorado, États-Unis, 9-13 octobre.

Caudal G. et D. Hauser, 1996 : Sea spectrum directional spreading function at short scale inferred from multi-frequency radar observations. J. Geophys. Res., 101(C7), 16, 601-613.

Chanzy A., T. J. Schmugge, J.-C. Calvet, Y. H. Kerr, P. Van Oevelen, O. Grosjean, J. R. Wang, 1997 : Airborne microwave radiometry on a semi arid area during HapexSahel. J. Hydrol., 188-189, 285-309.

Chepfer H., R. Valentin, V. Trouillet, G. Brogniez, P.-H. Flamant, J. Pelon et Y. Fouquart, 1996 : Determination of a radiatively equivalent microphysic for cirrus cloud during EUCREX'94 - Sensibility of radiative fluxes to the microphysic. Proceedings of the International Radiation Symposium, Fairbanks, Alaska, États-Unis, 19-24 août.

Coelho A., 1996 : Mesure granulométrique aéroportée dans les nuages. Thèse de l'université Paul Sabatier, Toulouse.

Coelho A., J.-L. Brenguier et T. Bourrianne, 1996 : A model for the Fast FSSP operation. 12th Int. Conf. on Clouds and Precip. Zurich, Suisse, août 1996, 458-461.

Delahaye J.-Y. et J. Lavergnat, 1994 : Mesure de l'humidité de l'air à l'aide d'un réfractomètre. Bulletin BNM, 98, 65-72.

Deschamps P.-Y., F.-M. Bréon, M. Leroy, A. Podaire, A. Bricaud, J.-C. Buriez et G. Sèze, 1994 : The POLDER mission: Instrument characteristics and scientific objectives. IEEE Trans. Geosci..Remote Sens., 32, 598-615.

Descloitres J., F. Parol et J.-C. Buriez, 1994 : On the validity of the plane-parallel approximation for cloud reflectances as measured from POLDER during ASTEX. Ann. Geophys., 13, 108-110.

Descloitres J., H. Pawlowska, J. Pelon, J.-L. Brenguier, F. Parol, J.-C. Buriez et P. Flamant, 1996 : Experimental retrieval of cloud optical thickness during EUCREX: comparison of three approaches. 12th Int. Conf. on Clouds and Precip. Zurich, Suisse, août 1996, 394-397. 
Deuzé J.-L., P. Goloub, M. Herman, P.-Y. Deschamps et R. Frouin, 1992 : Retrieval aerosols over the Gerlache Strait from aircraft photopolarimetric observations. Antarctic J., 27, 197-199.

Deuzé J.-L., F.-M. Bréon, P.-Y. Deschamps, C. Devaux, M. Herman, A. Podaire et J.-L. Roujean, 1993 : Analysis of the POLDER (POLarization and Directionality of Earth's Reflectances) airborne instrument observations over land surfaces. Remote Sens. Environ., 45, 137-154.

Druihet A., J.-L. Attié, L. de Sa Abreu, P. Durand et B. Benech, 1994 : Experimental study of inhomogeneous turbulence in the lower troposphere by wavelets analysis. Dans Wavelets Analysis and its Application. Edit. C. K. Chui, L. Montefusco et L. Puccio, Academic Press, New York, États-Unis, 543-559.

Druilhet A., C. Delon, R. Delmas, J.-P. Valette, B. Sinardet et N. Grand, 1996 : Mesure par avion des flux de constituants en trace de l'atmosphère à l'aide d'une méthode de prélèvement conditionnel. Atelier «Instrumentation et expérimentation », Toulouse, octobre 1996.

Dupont E., J. Pelon et C. Flamant, 1994 : Study of the moist convective atmospheric boundary layer structure by backscatter lidar. Bound.-Layer Meteor., 69, 1.

Eymard L., S. Planton, P. Durand, C. Le Visage, P.-Y. Le Traon, L. Prieur, A. Weill, D. Hauser, J. Rolland, J. Pelon, F. Baudin, B. Benech, J.-L. Brenguier, G. Caniaux, P. De Mey, E. Dombrowski, A. Druilhet, H. Dupuis, B. Ferret, C. Flamant, P. Flamant, F. Hernandez, D. Jourdan, K. Katsaros, D. Lambert, J.-M. Lefèvre, P. Le Borgne, B. Le Squère, A. Marsouin, H. Roquet, J. Tournadre, V. Trouillet, A. Tychensky et B. Zakardjian, 1996 : Study of the air-sea interactions at the mesoscale: the SEMAPHORE experiment. Ann. Geophys., 14, 986-1015.

Febvre G., 1994 : Lidar data inversion for cirrus clouds: An approach based on statistical analysis of in situ microphysical measurements. J. Atmos. Ocean. Technol., 11, 1231-1241.

Flamant C. et J. Pelon, 1996 : Atmospheric boundary layer structure over the Mediterranean sea during a Tramontane event. Quart. J. Roy. Meteor. Soc., 122, 1741-1778.

Frouin R., 1992 : Near-surface phytoplankton pigment concentration in the Gerlache Strait derived from aircraft POLDER data. Antarctic J., 27, 205-208.

Gayet J.-F., P. R. Brown et F. Albers, 1993 : A comparison on in-cloud measurements obtained with six PMS 2D-C probes. J. Atmos. Ocean. Technol., 10, 180-194.

Gayet J.-F., G. Febvre, G. Brogniez, H. Chepfer, W. Renger et P. Wending, 1996a : Microphysical and optical properties of cirrus and contrails. Cloud field study on 13 October 1989. J. Atmos. Sci., 53, 126-138.

Gayet J.-F., G. Febvre et H. Larsen, 1996b : On the reliability of the PMS FSSP probe in the presence of small ice crystals. J. Atmos. Ocean. Technol., 13, 1300-1310.

Gervaise C. et A. Loubet, 1994 : Validation de l'utilisation d'un réfractomètre à mesures rapides. Projet de fin d'études de l'École nationale supérieure d'ingénieurs de constructions aéronautiques, 88 pages.

Goloub P., J.-L. Deuzé, M. Herman et Y. Fouquart, 1994 : Analysis of the POLDER polarization measurements performed over cloud covers. IEEE Trans. Geosci. Remote Sens., 32, 78-88.

Goutorbe J.-P., T. Lebel, A. Tinga, P. Bessemoulin, J. Brouwer, A. J. Dolman, E. T. Engman, J. H. C. Gash, M. Hoepffner, P. Kabat, Y. H. Kerr, B. Monteny et S. Prince, 1994 : Hapex-Sahel: a large scale study of land-atmosphere interactions in the semi-arid tropics. Ann. Geophys., 12, 53-64

Greenberg J., A. Guenter, L. Klinger, W. Baugh, P. Harley, A. Druilhet et R. Delmas, 1997 : Biogenic VOCs and emissions in Central and Southern Africa: Results from EXPRESSO. AGU Fall Meeting, San Francisco, États-Unis, 8-12 décembre 1997. American Geophysical Union, vol. 78, n 46.

Grosjean O. et A. Sand, 1994 : Étalonnage du radiomètre hyperfréquence PORTOS et méthodes de correction des mesures. Dans Proc. 6th Int. Symp. Physical Measurements and Signatures in Remote Sensing, Val-d'Isère, France. Cnes, Toulouse, 513-518.

Hauser D., 1994 : Relationship between the direction of friction velocity and the direction of wind in various sea-state situations observed during the SEMAPHORE campaign. Preprints of the Second International Conference on Air-Sea Interaction and Meteorology and Oceanography of the Coastal zone, AMS, Lisbonne, Portugal, 257-258. 
Hauser D., G. Caudal, G. J. Rijckenberg, D. Vidal-Madjar, G. Laurent et P. Lancelin, 1992 : A new airborne FM/CW radar ocean wave spectrometer. IEEE Trans. Geosci. Remote Sens., 30 (5), 981-995.

Hauser D., G. Caudal et L. K. Shay, 1995 : Behaviour of the ocean radar-cross-section at low incidence, observed in the vicinity of the Gulf-Stream. IEEE Trans. Geosci. Remote Sens., 33, 1, 162-171.

Hauser D. et G. Caudal, 1996 : Combined analysis of the radar cross-section modulation due to the long ocean waves around 14 and $34^{\circ}$ incidence: implication for the hydrodynamic modulation. J. Geophys. Res., 101, C11, 25833-25846.

Herman M., J.-L. Deuzé, C. Devaux, F.-M. Bréon et D. Tanré, 1997 : Remote sensing of aerosols over land surfaces, including polarization measurements: application to some airborne POLDER measurements. J. Geophys. Res., sous presse.

Hicks B. B. et R. T. McMillen, 1984 : A simulation of the eddy method to measuring pollutant fluxes. J. Climate Appl. Meteor., 2, 637-643.

Kerr Y. H. et E. G. Njoku, 1990 : A semi-empirical model for interpreting microwave emission from semi-arid land surfaces as seen from space. IEEE Trans. Geosci. Remote Sens., 28, 384-393.

Kerr Y. H. et J.-P. Wigneron, 1995 : Vegetation models and observations: A review. Dans Passive microwave remote sensing of land-atmosphere interactions. Éd. B. J. Choudhury, Y. H. Kerr, E. G. Njoku et P. Pampaloni, VSP, 317-344.

Larsen H. R., J.-F. Gayet, G. Febvre, H. Chepfer et G. Brogniez, 1997 : Measurement errors in cirrus cloud microphysical properties. Accepté dans Ann. Geophys.

Lefèvre J.-M., B. Fradon, D. Le Meur, H. Roquet, B. Chapron et D. Hauser, 1994 : Surface sea-state modelling during the SEMAPHORE experiment. Preprints of the Second International Conference on Air-Sea Interaction and Meteorology and Oceanography of the Coastal zone, AMS, Lisbonne, Portugal, 130-131.

Leroy M. et F.-M. Bréon, 1996 : Angular signatures of surface reflectances from airborne POLDER data. Remote Sens. Environ., 57, 97-107.

Lohou F., A. Druilhet, P. Foster, B. Campistron, C. Gervaise, A. Loubet et J.-Y. Delahaye, 1995 : Measurement of $\mathrm{Cn}^{2}$ in the lower atmosphere with airborne refractometer. Comparison with simultaneous Doppler radar observations. 27th Conference on Radar Meteorology, Vail, Colorado, États-Unis, 9-13 octobre, 290-292.

Parol F., P. Goloub, J. Descloitres, J. Pelon et P. Flamant, 1996 : Comparison between four independant methods of cloud pressure derivation using POLDER and lidar measurements during EUCREX'94. Proc. of the International Radiation Symposium, Fairbanks, Alaska, États-Unis, 19-24 août.

Pawlowska H. et J.-L. Brenguier, 1996 : A study of the microphysical structure of stratocumulus clouds. 12th Int. Conf. on Clouds and Precip. Zurich, Suisse, août 1996, 23-26.

Pawlowska H., J.-L. Brenguier et G. Salut, 1997 : Optimal non-linear estimation for cloud particle measurements, J. Atmos. Ocean. Technol., 14, 88-104.

Pawlowska H., J.-L. Brenguier et F. Burnet, 1998 : Microphysical properties of stratocumulus clouds. Soumis à J. Atmos. Res.

Prince S. D., Y. H. Kerr, J.-P. Goutorbe, T. Lebel, A. Tinga, P. Bessemoulin, J. Brouwer, A. J. Dolman, E. T. Engman, J. H. C. Gash, M. Hoepffner, P. Kabat, B. Monteny, F. Saïd, P. Sellers et J. Wallace, 1995 : Geographical, biological and remote sensing aspects of the Hydrologic Atmospheric Pilot Experiment in the Sahel (HAPEX-Sahel). Remote Sens. Environ., 51, 215-234.

Quaglia P., D. Bruneau, A. Abchiche, M. Lopez, F. Fassina, J.-P. Marcovici, P. Genau, T. Danguy, B. Bient, B. Romand, C. Loth, M. Meissonnier, P.-H. Flamant et J. Pelon, 1996 : The airborne water vapor lidar LEANDRE 2: design, tests and first validations. Proc. of the 18th ILRC, Berlin. Ed. A. Ansmann, R. Neuber, P. Rairoux et U. Wandinger, Springer Verlag.

Quante M., P. R. A. Brown, R. Baumann, B. Guillemet et P. Hignett, 1996 : Three aircraft intercomparisons of dynamical and thermodynamical measurements during the PreEUCREX campaign. Contrib. Atmos. Phys., 69, 129-146.

Raju S., A. Chanzy, J.-P. Wigneron, J.-C. Calvet, Y. H. Kerr et L. Laguerre, 1995 : Soil moisture and temperature profile effects on microwave emission at low frequencies. Remote Sens. Environ., 85-97. 
Riom J. et D. Guyon, 1992 : Programme HRG (HRO), évaluation de simulations radiométriques pour les études sur la forêt. Cas de la forêt de pins maritimes des Landes de Gascogne. Rapport interne du Cnes, janvier 1992.

Sherjal I. et M. Fily, 1994 : Expérience de radiométre micro-onde sur la Vallée blanche, massif du Mont-Blanc. Neige et Avalanche, 68, 23-25.

Ström J., R. Busen, M. Quante, B. Guillemet, P. R. A. Brown et J. Heintzenberg, 1994 : Pre-EUCREX intercomparison of airborne humidity measuring instruments. J. Atmos. Ocean. Technol., 11, 1392-1399.

Van de Hulst H. C., 1957 : Light scattering by small particles. Wiley, New York, ÉtatsUnis.

Weill A., F. Baudin, H. Dupuis, L. Eymard, J.-P. Frangi, E. Gérard, P. Durand, B. Benech, J. Dessens, A. Druilhet, A. Rechou, P. Flamant, S. Elouragini, R. Valentin, G. Seze, J. Pelon, C. Flamant, J.-L. Brenguier, S. Planton, J. Rolland, A. Brisson, J. Le Borgne, A. Marsouin, T. Moreau, K. Katsaros, R. Monis, P. Queffeulou, J. Tournadre, P. K. Taylor, E. Kent, R. Pascal, P. Schibler, F. Parol, J. Descloitres, J.-Y. Balois, M. André et M. Charpentier, 1995 : SOFIA 1992 experiment during ASTEX. The Global Atmosphere and Ocean System, 3, 355-395.

Wigneron J.-P., J.-C. Calvet, A. Chanzy, O. Grosjean et L. Laguerre, 1995 : A composite Discrete Continuous approach to model the microwave emission of vegetation. IEEE Trans. Geosci. Remote Sens., 33, 1, 201-211.

Wigneron J.-P., J.-C. Calvet et Y. H. Kerr, 1996 : Monitoring water interception by crop fields from passive microwave observations. Agric. Forest Meteor., 80, 177-194.

Wigneron J.-P., Y. H. Kerr et L. Prévot, 1997 : Retrieval of soil and vegetation features from passive microwave measurements, Remote Sens. Environ., Reviews, 15, 157-177. 